\title{
MODULE STRUCTURE ON LIE POWERS AND NATURAL COALGEBRA-SPLIT SUB HOPF ALGEBRAS OF TENSOR ALGEBRAS
}

\author{
J. LI*, F. LEI, AND J. WU ${ }^{\dagger}$
}

\begin{abstract}
In this article, we investigate the functors from modules to modules that occur as the summands of tensor powers and the functors from modules to Hopf algebras that occur as natural coalgebra summands of tensor algebras. The main results provide some explicit natural coalgebra summands of tensor algebras. As a consequence, we obtain some decompositions of Lie powers over the general linear groups.
\end{abstract}

\section{INTRODUCTION}

The algebraic question for functorial coalgebra decompositions of the tensor algebras was arising from homotopy theory described as follows. The relevant topological question is how to decompose the loop spaces. This is a classical problem in homotopy theory with applications to homotopy groups. For instance, the classical results of Cohen-Moore-Neisendorfer [6] on the exponents of the homotopy groups of the spheres and Moore spaces were obtained from the study of the decompositions of the loop spaces of Moore spaces. The decompositions of the loop space functor $\Omega$ from $p$-local simply connected co- $H$-spaces to spaces was then introduced in 22] with the subsequent development in [20, 21, 24, 26]. Namely one gets the natural decompositions $\Omega X \simeq \bar{A}(X) \times \bar{B}(X)$ for some homotopy functors $\bar{A}$ and $\bar{B}$ on $p$-local simply-connected co- $H$-spaces $X$. Such decompositions may lose some information for an individual space $X$ in the sense that the functor $\bar{A}$ may be indecomposable but the space $\bar{A}(X)$ may have further decompositions. However the functorial decompositions has a good property that one can freely change the co- $H$-spaces $X$ in the decomposition formulas because they are functorial. Also there are examples of spaces $X$ such as Hopf invariant on complexes given in [12] with the property that the space $\bar{A}(X)$ is indecomposable for certain functor $\bar{A}$. A fundamental question concerning the functorial decompositions is how to determine the homology of the factors $\bar{A}(X)$ and $\bar{B}(X)$, which can be reduced to be a pure algebraic question as follows:

Let $V$ be any module over a field $\mathbf{k}=\mathbb{Z} / p$ and let $T(V)$ be the tensor algebra on $V$. Then $T(V)$ becomes a Hopf algebra by saying $V$ to be primitive. By

\footnotetext{
2000 Mathematics Subject Classification. Primary 55P35, 16W30, 17B60; Secondary 20C30, $55 \mathrm{P} 65$.

Key words and phrases. tensor algebras, Lie powers, coalgebras, sub Hopf algebras, $T_{n^{-}}$ projective functors, natural coalgebra decompositions.

*Partiall supported by the Academic Research Fund from Shijiazhuang Railway Institute.

$\dagger$ Research is supported in part by the Academic Research Fund of the National University of Singapore R-146-000-101-112.
} 
forgetting the algebra structure on $T(V)$, we have the functor $T$ from modules to coalgebras. Let

$$
T(V) \cong A(V) \otimes B(V)
$$

be any natural coalgebra decomposition of $T(V)$ for some functors $A$ and $B$ from (ungraded) modules to coalgebras. From [22, Theorem 1.3], the functors $A$ and $B$ can be canonically extended as functors from graded modules to graded coalgebras and the above decomposition formula holds for any graded module $V$. Then from [21, Theorem 1.1], the functors $A$ and $B$ induce functor $\bar{A}$ and $\bar{B}$ from co- $H$-spaces to spaces with the natural decomposition $\Omega X \simeq \bar{A}(X) \times \bar{B}(X)$ with the property that there exist filtrations on mod $p$ homology $H_{*}(\bar{A}(X))$ and $H_{*}(\bar{B}(X))$ such that its associated graded modules $E^{0} H_{*}(\bar{A}(X)) \cong A\left(\Sigma^{-1} \bar{H}_{*}(X)\right)$ and $E^{0} H_{*}(\bar{B}(X)) \cong B\left(\Sigma^{-1} \bar{H}_{*}(X)\right)$, where $\Sigma^{-1}$ is the desuspension of the graded modules. Briefly speaking, any coalgebra decomposition of the functor $T$ as in formula (1.1) induces a natural decomposition of the loops on $p$-local simply connected co- $H$-spaces in which the homology of its factors can be determined by their corresponding algebraic functors.

The functors $A$ and $B$ in decomposition (1.1) are complementary to each other and so it suffices to understand one of them as a coalgebra summand of the functor $T$. There exist some important coalgebra summands of $T$ in [22, Theorem 6.5] that give the functorial version of the Poincaré-Birkhoff-Witt Theorem. One of such functors is the functor $A^{\mathrm{min}}$, which is the smallest natural coalgebra summand of $T(V)$ containing $V$. The coalgebra complement of the functor $A^{\mathrm{min}}$, denoted by $B^{\max }$, has the property that $B^{\max }(V)$ can be chosen as a sub Hopf algebra of $T(V)$. However the determination of $A^{\min }(V)$ and $B^{\max }(V)$ seems out of current technology. As a consequence, the homology of the geometric realization $\bar{A}^{\min }(X)$ and $\bar{B}^{\max }(X)$ remains unknown. It is then important to find coalgebra summands $B$ of $T$ with the explicit information on $B(V)$ because in such a case the homology of the geometric realization $\bar{B}(X)$ can be understood.

The purpose of this article is to provide some explicit coalgebra summands $B$ of $T$. We are interested in the special cases where $B$ can be chosen as a sub Hopf algebra of $T$. This will give a relatively large coalgebra summand of $T$ because any subalgebra of tensor algebra is a tensor algebra, and so the complement functor $A$ of $B$ as in decomposition (1.1) becomes relatively small. Let $L_{n}(V)$ be the $n$th free Lie power on $V$, namely $L_{n}(V)$ is the homogenous component of the free Lie algebra $L(V)$ on $V$ of tensor length $n$. Our main result is as follows:

Theorem 1.1. Let the ground ring be a field of characteristic p. Let $\left\{m_{i}\right\}_{i \in I}$ be finite or infinite set of positive integers prime to $p$ with each $m_{i}>1$. Then the sub Hopf algebra of $T(V)$ generated by

$$
L_{m_{i} p^{r}}(V) \quad \text { for } i \in I, r \geq 0
$$

is a natural coalgebra summand of $T(V)$. In particular, the sub Hopf algebra $B(V)$ of $T(V)$ generated by

$$
L_{n}(V) \quad \text { for } n \text { NOT a power of } p
$$

is a natural coalgebra summand of $T(V)$.

By the maximum property of the functor $B^{\max }$, the sub Hopf algebras in the theorem are all contained in $B^{\max }$. According to [22, Proposition 11.1] as well as [4, 
the indecomposable elements in $B^{\max }$ does not has tensor length $p$ for $p>2$ and so the sub Hopf algebra $B(V)$ coincides with $B^{\max }(V)$ up to tensor length $p^{2}-1$. For the case $p=2$, the sub Hopf algebra $B(V)$ coincides with $B^{\max }(V)$ up to tensor length 7 according to the computations in 25. Our sub Hopf algebra $B(V)$ is strictly smaller than $B^{\max }(V)$ for general module $V$. However if the functors $B$ and $B^{\max }$ are extended to the functors from graded modules to graded modules in the sense of [22, the sub Hopf algebra $B(V)$ coincides with $B^{\max }(V)$ for graded modules $V$ of dimension $\leq p-1$ with $V_{\text {even }}=0$ according to [31, Theorem 1.1].

There is a canonical connection between coalgebra decompositions of $T$ and the decompositions of the Lie powers $L_{n}(V)$ as modules over the general linear groups by restricting decomposition (1.1) to the primitives. The decompositions of Lie powers have been actively studied in the recent development of representation theory [2, 3, 4, 9, 10].

The article is organized as follows. In section 2 we investigate the sub-quotient functors of the tensor power functors $T_{n}: V \mapsto T_{n}(V)=V^{\otimes n}$ from modules to modules. These special functors are of course closely related to the tensor representation of the symmetric groups and the finite dimensional polynomial representations of the general linear groups (by evaluating on a fixed module $V$ ). They are also related the modules over the Schur algebras and the modules over the Steenrod algebra [15, 16, 17]. In this section, we introduce exact functors $\gamma_{n}(-)$ from the category of functors from modules to modules to the category of the modules over the symmetric groups in this section which are variations of the classical Schur functor [1, 19, 11. In geometry, the summands of the tensor power functors $T_{n}$ are closely related to decompositions of self-smash products [23, 29].

In section 3. we investigate the subfunctors of the Lie powers $L_{n}$ that occur as the summands of the functor $T_{n}$, which we call $T_{n}$-projective subfunctors of $L_{n}$. According to Theorem 3.9 these functors are closely related to the summands of the Lie powers $L_{n}(V)$ that occur as summands of $V^{\otimes n}$ studied in [3, 9, 10.

We give a coalgebra decomposition of $T$ called the Block Decomposition in section 4 . According to Theorem 4.3, this is a coalgebra decomposition of $T$ in the form

$$
T \cong \bigotimes_{i=1}^{\infty} C^{m_{i}}
$$

where $\left\{m_{i}\right\}$ is the set of all positive integers prime to $p$ and the primitives of $C^{m_{i}}$ are exactly given by the primitives of the tensor algebra $T$ with tensor length $m_{i} p^{r}$ for $r \geq 0$. In other words, the primitives of the tensor algebra $T$ with tensor length $m p^{r}$ for $r \geq 0$ for each $m$ prime to $p$ can be blocked into a coalgebra summand $C^{m}$ of $T$.

The proof of Theorem 1.1 is given in section 5 In section 6 we give some applications of our decomposition theorem to Lie powers by restricting to the primitives.

\section{The structure on the Tensor Powers}

2.1. Tensor Algebras. Let $V$ be any module and let

$$
T(V)=\bigoplus_{n=0}^{\infty} V^{\otimes n}
$$

be the tensor algebra generated by $V$, where $V^{\otimes n}=\mathbf{k}$ and the multiplication on $T(V)$ is given by the formal tensor product of monomials. The tensor algebra 
admits the universal property that, for any associated algebra $A$ and any linear map $f: V \rightarrow A$, there exists a unique algebra map $\tilde{f}: T(V) \rightarrow A$ such that $\left.\tilde{f}\right|_{V}=f$. In particular, the linear map

$$
V \longrightarrow T(V) \otimes T(V) \quad x \mapsto x \otimes 1+1 \otimes x
$$

extends uniquely to an algebra map $\psi: T(V) \rightarrow T(V) \otimes T(V)$ and so $T(V)$ has the canonical Hopf algebra structure with the multiplication given by the formal tensor product of monomials and the comultiplication given by $\psi$. We refer to [18] as a classical reference for Hopf algebras and quasi-Hopf algebras. The comultiplication $\psi$ is coassociative and cocommutative. The module $T(V)$ with the comultiplication $\psi: T(V) \rightarrow T(V) \otimes T(V)$ is called shuffle coalgebra as its graded dual

$$
T^{*}(V)=\bigoplus_{n=0}^{\infty}\left(V^{\otimes n}\right)^{*} \cong \bigoplus_{n=0}^{\infty}\left(V^{*}\right)^{\otimes n}
$$

is the usual shuffle algebra under the multiplication $\psi^{*}: T^{*}(V) \otimes T^{*}(V) \rightarrow T^{*}(V)$.

We are interested in the functor $T: V \mapsto T(V)$. There are three terminologies on this functor:

(1). The functor $T^{H}: V \mapsto T(V)$ from modules to Hopf algebras;

(2). The functor $T^{C}: V \mapsto T(V)$ from modules to coalgebras by forgetting the multiplication;

(3). The functor $T^{M}: V \mapsto T(V)$ from modules to modules by forgetting both multiplication and comultiplication.

The notation of the functor $T$ refers one of $T^{H}, T^{C}$ or $T^{M}$ if the working category is clear.

By taking the tensor length from the definition of $T(V)$, the functor $T^{M}$ admits a natural decomposition that

$$
T^{M} \cong \bigoplus_{n=0}^{\infty} T_{n}
$$

where $T_{n}(V)=V^{\otimes n}$ with $T_{0}(V)=\mathbf{k}$. Thus the functors $T^{H}, T^{C}$ and $T^{M}$ are graded functors. From the well-known property (see for instance [13, Lemma 3.8]) that

$$
\operatorname{Hom}\left(T_{n}, T_{m}\right)=\left\{\begin{array}{lll}
0 & \text { if } & n \neq m \\
\mathbf{k}\left(\Sigma_{n}\right) & \text { if } & n=m,
\end{array}\right.
$$

the decomposition of $T^{M}$ is in fact an orthogonal decomposition. A direct consequence is that the comultiplication $\psi$ (as a natural transformation) is uniquely determined by the multiplication on $T(V)$ for having the Hopf structure.

Proposition 2.1. Let $\Delta_{V}: T^{M}(V) \rightarrow T^{M}(V) \otimes T^{M}(V)$ be a natural transformation such that $T^{M}(V)$ with the usual multiplication together with the comultiplication given by $\Delta_{V}$ is a quasi-Hopf algebra for every $V$. Then $\Delta_{V}=\psi_{V}$ for all $V$.

Proof. For every $V$, from the property that $\operatorname{Hom}\left(T_{n}, T_{m}\right)=0$ for $n \neq m$, we have

$$
\Delta_{V}\left(T_{1}(V)\right) \subseteq T_{1}(V) \otimes T_{0}(V) \oplus T_{0}(V) \otimes T_{1}(V)
$$

and the counit

$$
\epsilon_{V}: T(V)=\bigoplus_{n=0}^{\infty} T_{n}(V) \longrightarrow T_{0}(V)
$$


is the canonical projection by sending each $T_{i}(V)$ to 0 for $i>0$ and $\left.\epsilon\right|_{T_{0}(V)}=\mathrm{id}$. Since both $\Delta_{V}$ and $\psi_{V}$ has the counit uniquely given by $\epsilon_{V}$, we have

$$
\left.\Delta_{V}\right|_{T_{1}(V)}=\left.\psi_{V}\right|_{T_{1}(V)}
$$

It follows that $\Delta_{V}=\psi_{V}$ because both of them are algebra map with respect to formal tensor product.

Remark 2.2. Given a module $V$, of course one could have many comultiplication on $T(V)$ such that $T(V)$ is Hopf. The proposition states that $\psi$ is the only one possible comultiplication on $T(V)$ as a natural transformation.

2.2. Subfunctors of the Tensor Algebra Functor. Let $\mathcal{C}$ and $\mathcal{D}$ be categories and let $A, B: \mathcal{C} \rightarrow \mathcal{D}$ be functors. We call $A$ is a subfunctor (quotient functor) of $B$ if there is a natural transformation $\phi: A \rightarrow B$ such that

$$
\phi_{X}: A(X) \rightarrow B(X)
$$

is injective (surjective) for every object $X \in \mathcal{C}$. A subfunctor (quotient functor) of $T$ refers to a subfunctor (quotient functor) of $T^{H}, T^{C}$ or $T^{M}$. A subfunctor (quotient functor) of $T^{H}$ is called a sub Hopf functor (quotient Hopf functor) of $T$. Similarly we have sub coalgebra functor (quotient coalgebra functor) of $T$ and submodule functor (quotient module functor) of $T$. A graded subfunctor (graded quotient functor) of $T$ refers to a subfunctor of $T^{H}, T^{C}$ or $T^{M}$ as functors from modules to graded Hopf algebras, graded coalgebras or graded modules, respectively.

Proposition 2.3. Let $B$ be a subfunctor of $T$. Then $B$ is a graded subfunctor of $T$.

Proof. Let $\phi_{V}: B(V) \rightarrow T(V)$ be the natural monomorphism and let

$$
B_{n}(V)=\phi_{V}^{-1}\left(T_{n}(V)\right)
$$

for any $V$. From the fact that $T=\bigoplus_{n=0}^{\infty} T_{n}$ is an orthogonal decomposition, the functor $B$ admits a graded decomposition

$$
B=\bigoplus_{n=0}^{\infty} B_{n}
$$

Thus if $B$ is a subfunctor of $T^{M}$, then $B$ is graded subfunctor of $T^{M}$. By the orthogonal property of $T$ as in equation (2.2),

$$
\operatorname{Hom}\left(B_{n}, B_{m}\right)=0
$$

for $n \neq m$. Thus if $B$ is a subfunctor of $T^{H}$ or $T^{C}$, then the multiplication and comultiplication on $B$ whence it is defined as a natural transformation must be graded and hence the result.

Corollary 2.4. Let $C$ be a quotient functor of $T$. Then $C$ is a graded quotient functor of $T$.

Proof. Let $B$ be the kernel of $T^{M} \rightarrow C$ by forgetting the possible Hopf or coalgebra structure on $C$. By Proposition 2.3, $B$ is a graded subfunctor of $T^{M}$ and so $C$ is a graded quotient functor of $T^{M}$, where $C_{n}=T_{n} / B_{n}$ for each $n \geq 0$. By the orthogonal property of $T$ as in equation (2.2),

$$
\operatorname{Hom}\left(C_{n}, C_{m}\right)=0
$$


for $n \neq m$. Thus if $C$ is a quotient functor of $T^{H}$ or $T^{C}$, then the multiplication and comultiplication on $C$ whence it is defined as a natural transformation must be graded and hence the result.

2.3. The Associated Symmetric Group Modules of the Functors. Let $\bar{V}_{n}$ be the $n$-dimensional $\mathbf{k}$-module with a fixed choice of basis $\left\{x_{1}, \ldots, x_{n}\right\}$. For each $1 \leq i \leq n$, define the linear transformation

$$
d_{i}: \bar{V}_{n} \longrightarrow \bar{V}_{n-1}
$$

by setting

$$
d_{i}\left(x_{j}\right)=\left\{\begin{array}{lll}
x_{j} & \text { if } & j<i, \\
0 & \text { if } & j=i, \\
x_{j-1} & \text { if } & j>i .
\end{array}\right.
$$

The right $\mathbf{k}\left(\Sigma_{n}\right)$-action on $\bar{V}_{n}$ is given by

$$
x_{i} \cdot \sigma=x_{\sigma(i)}
$$

for $1 \leq i \leq n$ and $\sigma \in \Sigma_{n}$. Then, for each $1 \leq i \leq n$ and any $\sigma \in \Sigma_{n}$, clearly there exists a unique permutation $d_{i} \sigma \in \Sigma_{n-1}$ such that the diagram

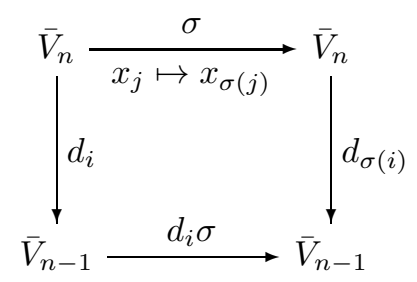

commutes. Let $B$ be a functor from modules to modules. Then $B\left(\bar{V}_{n}\right)$ is a right $\mathbf{k}\left(\Sigma_{n}\right)$-module induced by the action of $\mathbf{k}\left(\Sigma_{n}\right)$ on $\bar{V}_{n}$. Define

$$
\gamma_{n}(B)=\bigcap_{i=1}^{n} \operatorname{Ker}\left(B\left(d_{i}\right): B\left(\bar{V}_{n}\right) \rightarrow B\left(\bar{V}_{n-1}\right)\right) .
$$

By applying the functor $B$ to diagram (2.3),$\gamma_{n}(B)$ is a $\mathbf{k}\left(\Sigma_{n}\right)$-submodule of $B\left(\bar{V}_{n}\right)$. Let

$$
\phi: B \rightarrow C
$$

be a natural transformation of functors from modules to modules. Then clearly $\phi_{\bar{V}_{n}}$ induces a $\mathbf{k}\left(\Sigma_{n}\right)$-map

$$
\gamma_{n}(\phi): \gamma_{n}(B) \longrightarrow \gamma_{n}(C)
$$

Proposition 2.5. Let

$$
A \stackrel{j}{\longrightarrow} B \stackrel{p}{\longrightarrow} C
$$

be a short exact sequence of functors from modules to modules. Then there is a short exact sequence of $\mathbf{k}\left(\Sigma_{n}\right)$-modules

$$
\gamma_{n}(A) \stackrel{\gamma_{n}(j)}{\longrightarrow} \gamma_{n}(B) \stackrel{\gamma_{n}(p)}{\longrightarrow} \gamma_{n}(C) .
$$

Thus $\gamma_{n}(-)$ is an exact functor from the category of functors from modules to modules to the category of $\mathbf{k}\left(\Sigma_{n}\right)$-modules. 
Note. The exact functor $\gamma_{n}(-)$ is a variation of the Schur functor given in [1] in the following sense: Let $B$ is a sub-quotient functor of $T_{n}$ and let $V$ be a module with $m=\operatorname{dim} V \geq n$. Then $B(V)$ is a sub-quotient $\mathbf{k}\left(G L_{m}(\mathbf{k})\right)$-module of $V^{\otimes n}$. Let $\bar{V}_{n}$ embed into $V$ in the canonical way such that $V=\bar{V} \oplus V^{\prime}$. In our definition, $\gamma_{n}(B) \subseteq B(\bar{V}) \subseteq B(V)$. According to [9, Section 1.2, p.71], $B(V) \mapsto \gamma_{n}(B)$ is the Schur functor.

Proof. Define coface operation $d^{i}: \bar{V}_{n-1} \rightarrow \bar{V}_{n}$ by setting

$$
d^{i}\left(x_{j}\right)=\left\{\begin{array}{lll}
x_{j} & \text { if } & j<i \\
x_{j+1} & \text { if } & j \geq i
\end{array}\right.
$$

for $1 \leq i \leq n$. Then the sequence of module $\left\{\bar{V}_{n+1}\right\}_{n \geq 0}$ with faces

$$
d_{1}, \ldots, d_{n}: \bar{V}_{n} \rightarrow \bar{V}_{n-1}
$$

relabeled as $d_{0}, \ldots, d_{n-1}$ and cofaces

$$
d^{1}, \ldots, d^{n}: \bar{V}_{n-1} \rightarrow \bar{V}_{n}
$$

relabeled as $d^{0}, \ldots, d^{n-1}$ by shifting indices down by 1 forms a bi- $\Delta$-groups in the sense of [30, Section 1.2]. By applying the functors to the bi- $\Delta$-group $\left\{\bar{V}_{n+1}\right\}_{n \geq 0}$, one gets a short exact sequence of bi- $\Delta$-groups

$$
\left\{A\left(\bar{V}_{n+1}\right)\right\}_{n \geq 0} \longrightarrow\left\{B\left(\bar{V}_{n+1}\right)\right\}_{n \geq 0} \longrightarrow\left\{C\left(\bar{V}_{n+1}\right)\right\}_{n \geq 0} .
$$

The assertion then follows by [30, Proposition 1.2.10].

Corollary 2.6. Let $\phi: A \rightarrow B$ be a natural transformation between functors from modules to modules. Suppose that

$$
\gamma_{n}(\phi): \gamma_{n}(A) \rightarrow \gamma_{n}(B)
$$

is an isomorphism for each $n \geq 1$. Then

$$
\phi_{V}: A(V) \rightarrow B(V)
$$

is an isomorphism for any finite dimensional module $V$. Thus if both $A$ and $B$ preserve colimits, then $\phi$ is a natural equivalence.

Proof. Let $C$ be the cokernel of $\phi$. Suppose that $C(V) \neq 0$ for some finite dimensional module $V$. Let

$$
n=\min \{k \mid C(V) \neq 0 \operatorname{dim}(V)=k\} .
$$

Then $\gamma_{n}(C)=C\left(\bar{V}_{n}\right) \neq 0$. By Proposition 2.5, $\gamma_{n}(C)=0$ which is a contradiction. Thus $C(V)=0$ for any finite dimensional module $V$. Similarly, for $D$ the kernel of $\phi$, we have $D(V)=0$ for any finite dimensional module $V$, finishing the proof.

2.4. $T_{n}$-projective functors. Consider the functor $T_{n}$. Let $\gamma_{n}=\gamma_{n}\left(T_{n}\right)$. Then $\gamma_{n}$ is the k-submodule of $\bar{V}_{n}^{\otimes n}$ spanned by the monomials

$$
x_{\sigma(1)} \otimes \cdots \otimes x_{\sigma(n)}
$$

for $\sigma \in \Sigma_{n}$ with the right symmetric group action explicitly given by

$$
\left(x_{i_{1}} \otimes \cdots \otimes x_{i_{n}}\right) \cdot \sigma=x_{\sigma\left(i_{1}\right)} \otimes \cdots \otimes x_{\sigma\left(i_{n}\right)}
$$

for $\sigma \in \Sigma_{n}$ and the monomials $x_{i_{1}} \cdots x_{i_{n}} \in \gamma_{n}$. Observe that

$$
\gamma_{n} \cong \mathbf{k}\left(\Sigma_{n}\right)
$$

as a $\mathbf{k}\left(\Sigma_{n}\right)$-module. 
Let $V$ be any k-module and let $a_{1}, \ldots, a_{n} \in V$. We write $a_{1} \cdots a_{n}$ for the tensor product $a_{1} \otimes \cdots \otimes a_{n} \in V^{\otimes n}$ if there are no confusions. Let the symmetric group $\Sigma_{n}$ act on $V^{\otimes n}$ by permuting positions. More precisely the left $\mathbf{k}\left(\Sigma_{n}\right)$-action on $V^{\otimes n}$ is given

$$
\sigma \cdot\left(a_{1} \cdots a_{n}\right)=a_{\sigma(1)} \cdots a_{\sigma(n)}
$$

for $\sigma \in \Sigma_{n}$ and the monomials $a_{1} \cdots a_{n} \in V^{\otimes n}$. Let $B$ be any functor from modules to modules. Define the functor $\gamma_{n}^{B}(-)$ by setting

$$
\gamma_{n}^{B}(V)=\gamma_{n}(B) \otimes_{\mathbf{k}\left(\Sigma_{n}\right)} V^{\otimes n}
$$

for any module $V$. Clearly

$$
\gamma_{n}^{T_{n}}(V)=\gamma_{n} \otimes_{\mathbf{k}\left(\Sigma_{n}\right)} V^{\otimes n} \cong T_{n}(V) .
$$

Proposition 2.7. Let

$$
B \stackrel{j}{\longrightarrow} T_{n} \stackrel{p}{\longrightarrow} C C
$$

be a short exact sequence of functors from modules to modules. Then the natural isomorphism $\gamma_{n} \otimes_{\mathbf{k}\left(\Sigma_{n}\right)} V^{\otimes n} \cong T_{n}(V)$ induces a natural commutative diagram of exact sequences

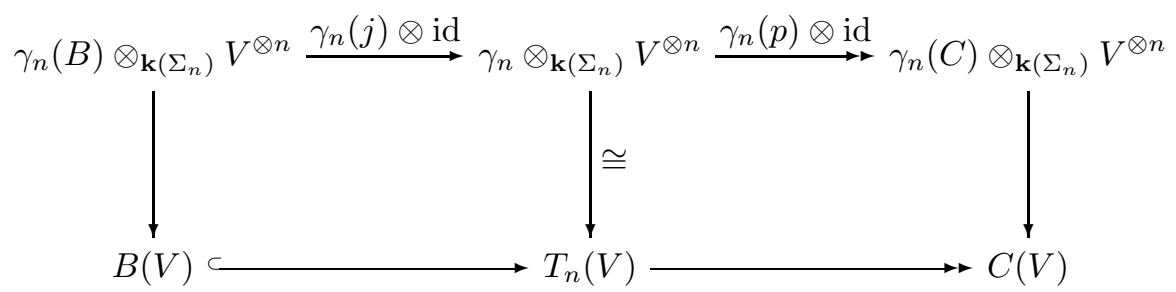

with a natural exact sequence

$\operatorname{Tor}_{1}^{\mathbf{k}\left(\Sigma_{n}\right)}\left(\gamma_{n}(C), V^{\otimes n}\right) \hookrightarrow \gamma_{n}(B) \otimes_{\mathbf{k}\left(\Sigma_{n}\right)} V^{\otimes n} \rightarrow B(V) \rightarrow \gamma_{n}(C) \otimes_{\mathbf{k}\left(\Sigma_{n}\right)} V^{\otimes n} \rightarrow C(V)$. for any module $V$.

Proof. By taking the image of $j_{V}$, we may consider $B(V)$ is a submodule of $T_{n}(V)$ for any module $V$. Let

$$
\theta: \gamma_{n} \otimes_{\mathbf{k}\left(\Sigma_{n}\right)} V^{\otimes n} \rightarrow T_{n}(V)
$$

be the isomorphism and let $\Phi_{V}^{B}=\theta \circ\left(\gamma_{n}(j) \otimes\right.$ id $)$. Observe that the isomorphism $\theta$ is given by

$$
\theta\left(x_{1} \cdots x_{n} \otimes a_{1} \cdots a_{n}\right)=a_{1} \cdots a_{n}
$$

for $a_{1}, \ldots, a_{n} \in V$. Let $a=a_{1} \cdots a_{n} \in V^{\otimes n}$ be any monomial with $a_{j} \in V$ for $1 \leq j \leq n$ and let

$$
\alpha=\sum_{\sigma \in \Sigma_{n}} k_{\sigma} x_{\sigma(1)} \cdots x_{\sigma(n)} \in \gamma_{n}(B) .
$$

Then

$$
\begin{aligned}
\Phi_{V}^{B}\left(\alpha \otimes a_{1} \cdots a_{n}\right) & =\sum_{\sigma \in \Sigma_{n}} k_{\sigma} x_{\sigma(1)} \cdots x_{\sigma(n)} \otimes a_{1} \cdots a_{n} \\
& =\sum_{\sigma \in \Sigma_{n}} k_{\sigma}\left(x_{1} \cdots x_{n}\right) \cdot \sigma \otimes a_{1} \cdots a_{n} \\
& =\sum_{\sigma \in \Sigma_{n}} k_{\sigma} x_{1} \cdots x_{n} \otimes \sigma \cdot\left(a_{1} \cdots a_{n}\right) \\
& =\sum_{\sigma \in \Sigma_{n}} k_{\sigma} x_{1} \cdots x_{n} \otimes a_{\sigma(1)} \cdots a_{\sigma(n)} \\
& =\sum_{\sigma \in \Sigma_{n}} k_{\sigma} a_{\sigma(1)} \cdots a_{\sigma(n)} \in V^{\otimes n}
\end{aligned}
$$


Define a linear transformation $f_{a}: \bar{V}_{n} \rightarrow V$ by setting

$$
f_{a}\left(x_{i}\right)=a_{i}
$$

for $1 \leq i \leq n$. Consider $T_{n}\left(f_{a}\right)=f_{a}^{\otimes n}: T_{n}\left(\bar{V}_{n}\right) \rightarrow T_{n}(V)$. Then

$$
T_{n}\left(f_{a}\right)(\alpha)=f_{a}^{\otimes n}\left(\sum_{\sigma \in \Sigma_{n}} k_{\sigma} x_{\sigma(1)} \cdots x_{\sigma(n)}\right)=\Phi_{V}^{B}\left(\alpha \otimes a_{1} \cdots a_{n}\right) .
$$

From the commutative diagram

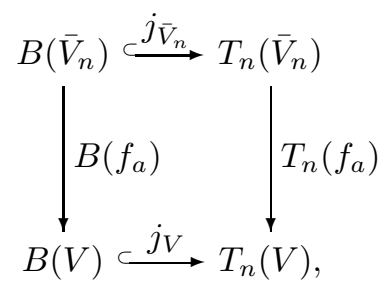

since

$$
\alpha \in \gamma_{n}(B) \subseteq B\left(\bar{V}_{n}\right)
$$

we have

$$
\Phi_{V}^{B}\left(\alpha \otimes a_{1} \cdots a_{n}\right)=T_{n}\left(f_{a}\right)(\alpha) \in B(V) .
$$

It follows that

$$
\operatorname{Im}\left(\Phi_{V}^{B}\right) \subseteq B(V)
$$

for any module $V$. Thus we have the left square commutative diagram in the statement.

By Proposition 2.5, there is a short exact sequence of $\mathbf{k}\left(\Sigma_{n}\right)$-modules

$$
\gamma_{n}(B) \stackrel{\gamma_{n}(j)}{\longrightarrow} \gamma_{n} \stackrel{\gamma_{n}(p)}{\longrightarrow} \gamma_{n}(C) .
$$

Since $\gamma_{n}$ is a free $\mathbf{k}\left(\Sigma_{n}\right)$-module, there is a exact sequence

$\operatorname{Tor}_{1}^{\mathbf{k}\left(\Sigma_{n}\right)}\left(\gamma_{n}(C), V^{\otimes n}\right) \hookrightarrow \gamma_{n}(B) \otimes_{\mathbf{k}\left(\Sigma_{n}\right)} V^{\otimes n} \rightarrow \gamma_{n} \otimes_{\mathbf{k}\left(\Sigma_{n}\right)} V^{\otimes n} \rightarrow \gamma_{n}(C) \otimes_{\mathbf{k}\left(\Sigma_{n}\right)} V^{\otimes n}$.

Hence we have the right square of the commutative diagram as well as the exact sequence in the statement.

Example 2.8. We give an example that the natural transformation

$$
\gamma_{n}(B) \otimes_{\mathbf{k}\left(\Sigma_{n}\right)} V^{\otimes n} \longrightarrow B(V)
$$

could be neither epimorphism nor monomorphism for subfunctors $B$ of $T_{n}$. Let $\mathbf{k}$ be a field of characteristic 2. The Lie power $L_{2}(V)$ is the submodule of $V^{\otimes 2}$ spanned by $[a, b]=a b-b a$ for $a, b \in V$. The restricted Lie power $L_{2}^{\text {res }}(V)$ is the submodule of $V^{\otimes 2}$ spanned by $a^{2},[a, b]$ for $a, b \in V$. Then

$$
\gamma_{2}\left(L_{2}\right)=\gamma_{2}\left(L_{2}^{\text {res }}\right)
$$

is the 1-dimensional submodule of $\mathbf{k}\left(\Sigma_{2}\right)$ generated by $1-\tau$. Since $\mathbf{k}$ is of characteristic $2, \gamma_{2}\left(L_{2}\right)=\gamma_{2}\left(L_{2}^{\text {res }}\right)$ is the trivial $\mathbf{k}\left(\Sigma_{2}\right)$-module and so

$$
\gamma_{2}\left(L_{2}^{\text {res }}\right) \otimes_{\mathbf{k}\left(\Sigma_{2}\right)} V^{\otimes 2}=S_{2}(V),
$$

the 2-fold symmetric product of $V$. The natural transformation

$$
\gamma_{2}\left(L_{2}^{\text {res }}\right) \otimes_{\mathbf{k}\left(\Sigma_{2}\right)} V^{\otimes 2} \longrightarrow L_{2}^{\text {res }}(V)
$$


is not epimorphism for $V$ with $\operatorname{dim} V \geq 1$ because its image is given by $L_{2}(V)$. The kernel of this natural transformation is measured by

$$
\operatorname{Tor}_{1}^{\mathbf{k}\left(\Sigma_{2}\right)}\left(\gamma_{2} / \operatorname{Lie}(2), V^{\otimes 2}\right) \neq 0
$$

for $V$ with $\operatorname{dim} V \geq 1$.

Let $B$ be a functor from modules to modules. The dual functor $B^{*}$ is defined as follows. For any finite dimensional module $V$, define

$$
B^{*}(V)=B\left(V^{*}\right)^{*},
$$

where $V^{*}=\operatorname{Hom}_{\mathbf{k}}(V, \mathbf{k})$ is the dual $\mathbf{k}$-module of $V$, and, for a general module $V$, let

$$
B^{*}(V)=\operatorname{colim}_{V_{\alpha}} B^{*}\left(V_{\alpha}\right)
$$

be the direct limit of the module $B^{*}\left(V_{\alpha}\right)$ subject to the direct system given by the diagram of all finite dimensional submodules of $V$ with inclusions. Clearly $T_{n}^{*}=T_{n}$.

Proposition 2.9. Let $B$ be a functor from modules to modules. Then $\gamma_{n}\left(B^{*}\right)$ is the dual $\mathbf{k}\left(\Sigma_{n}\right)$-module of $\gamma_{n}(B)$ for each $n \geq 1$.

Proof. For the basis $\left\{x_{1}, \ldots, x_{n}\right\}$ for $\bar{V}_{n}$, let $\left\{x_{1}^{*}, \ldots, x_{n}^{*}\right\}$ be the standard dual basis of $\bar{V}_{n}^{*}$. Let

$$
\theta_{n}: \bar{V}_{n} \rightarrow \bar{V}_{n}^{*}
$$

be the linear transformation such that $\theta_{n}\left(x_{j}\right)=x_{j}^{*}$ for $1 \leq j \leq n$. Then it is routine to check that the composite

$$
\gamma_{n}\left(B^{*}\right) \subseteq B^{*}\left(\bar{V}_{n}\right)=B\left(\bar{V}_{n}^{*}\right)^{*} \stackrel{B\left(\theta_{n}\right)^{*}}{\longrightarrow} B\left(\bar{V}_{n}\right)^{*} \longrightarrow \gamma_{n}(B)^{*}
$$

is an isomorphism of $\mathbf{k}\left(\Sigma_{n}\right)$-modules.

We call a direct sum of copies of $T_{n}$ a free $T_{n}$-functor. A functor $B$ from modules to modules is called $T_{n}$-projective if there exists a free $T_{n}$-functor $F$ together with natural transformations $s: B \rightarrow F$ and $r: F \rightarrow B$ such that $r \circ s: B \rightarrow B$ is a natural equivalence. In other words, a $T_{n}$-projective functor means a summand (or retract) of a free $T_{n}$-functor.

Proposition 2.10. Let $B$ be a functor from modules to modules.

1) If $B$ is a $T_{n}$-projective functor, then $\gamma_{n}(B)$ is a projective $\mathbf{k}\left(\Sigma_{n}\right)$-module and there is a natural isomorphism

$$
\gamma_{n}(B) \otimes_{\mathbf{k}\left(\Sigma_{n}\right)} V^{\otimes n} \cong B(V)
$$

for any module $V$.

2) If $B$ is a subfunctor of a direct sum of finite copies of $T_{n}$ with the property that $\gamma_{n}(B)$ is a projective $\mathbf{k}\left(\Sigma_{n}\right)$-module, then $B$ is a $T_{n}$-projective functor. Moreover there is a natural equivalence $B \cong B^{*}$.

3) If $B$ is a quotient functor of a direct sum of finite copies of $T_{n}$ with the property that $\gamma_{n}(B)$ is a projective $\mathbf{k}\left(\Sigma_{n}\right)$-module, then $B$ is a $T_{n}$-projective functor. Moreover there is a natural equivalence $B \cong B^{*}$.

4) Let $B$ be a sub-quotient functor of a direct sum of finite copies of $T_{n}$. Suppose that $B$ is a $T_{n}$-projective functor. Then $B$ is both projective and injective in the category of sub-quotient functors of direct sums of finite copies of $T_{n}$. 
Proof. The proof of assertion (1) is straightforward. Assertion (3) follows from (2) by considering the dual functor.

(2). Let $B$ be a subfunctor of $F$, where $F$ is a finite direct sum of copies of $T_{n}$. Let $C=F / B$. Then there is a short exact sequence

$$
\gamma_{n}(B) \hookrightarrow \gamma_{n}(F) \longrightarrow \gamma_{n}(C) .
$$

Since $\gamma_{n}(B)$ is a finitely generated projective $\mathbf{k}\left(\Sigma_{n}\right)$-module, it is an injective $\mathbf{k}\left(\Sigma_{n}\right)$ module and so the above short exact sequence splits off as $\mathbf{k}\left(\Sigma_{n}\right)$-modules. It follows that $\gamma_{n}(C)$ is a projective $\mathbf{k}\left(\Sigma_{n}\right)$-module because $\gamma_{n}(F)$ is a free $\mathbf{k}\left(\Sigma_{n}\right)$-module.

$$
\operatorname{Tor}_{1}^{\mathbf{k}\left(\Sigma_{n}\right)}\left(\gamma_{n}(C), V^{\otimes n}\right)=0 .
$$

Since $F$ is a direct sum of copies of the functor $T_{n}$, we can apply the exact sequence in Proposition 2.7 In particular, the natural transformation

$$
\Phi_{V}^{B}: \gamma_{n}(B) \otimes_{\mathbf{k}\left(\Sigma_{n}\right)} V^{\otimes n} \longrightarrow B(V)
$$

is a natural monomorphism. The natural inclusion $B \hookrightarrow F$ induces an natural epimorphism $F=F^{*} \rightarrow B^{*}$. By Proposition 2.7, there is a natural epimorphism

$$
\Phi_{V}^{B^{*}}: \gamma_{n}\left(B^{*}\right) \otimes_{\mathbf{k}\left(\Sigma_{n}\right)} V^{\otimes n} \longrightarrow B^{*}(V) \text {. }
$$

By Proposition 2.9,

$$
\gamma_{n}\left(B^{*}\right) \cong \gamma_{n}(B)^{*} \cong \gamma_{n}(B)
$$

as $\mathbf{k}\left(\Sigma_{n}\right)$-modules because $\gamma_{n}(B)$ is a finitely generated $\mathbf{k}\left(\Sigma_{n}\right)$-projective modules. Let $V$ be any finite dimensional $\mathbf{k}$-module. From equations (2.10) and (2.11, we have

$$
\operatorname{dim} B(V)=\operatorname{dim} B\left(V^{*}\right)^{*}=\operatorname{dim} B^{*}(V) \leq \operatorname{dim}\left(\gamma_{n}(B) \otimes_{\mathbf{k}\left(\Sigma_{n}\right)} V^{\otimes n}\right) \leq \operatorname{dim} B(V) .
$$

Thus $\Phi_{V}^{B}$ and $\Phi_{V}^{B^{*}}$ are isomorphisms for any finite dimensional module $V$. Since the functors $\gamma_{n}(B) \otimes_{\mathbf{k}\left(\Sigma_{n}\right)}(-)^{\otimes n}, B$ and $B^{*}$ preserve colimits, the natural transformations $\Phi^{B}$ and $\Phi^{B^{*}}$ are natural equivalences. The assertion now follows from the fact that $\gamma_{n}(B) \otimes_{\mathbf{k}\left(\Sigma_{n}\right)} V^{\otimes n}$ is a natural summand of $\gamma_{n}(F) \otimes_{\mathbf{k}\left(\Sigma_{n}\right)} V^{\otimes n} \cong F(V)$.

(4). Let $\mathcal{C}$ be the category of sub-quotient functors of direct sums of copies of $T_{n}$. It suffices to show that $T_{n}$ is projective and injective in the $\mathcal{C}$. Let $B$ be an object in $\mathcal{C}$ with a natural epimorphism $q: B \rightarrow T_{n}$. It induces an epimorphism $\gamma_{n}(q): \gamma_{n}(B) \rightarrow \gamma_{n}\left(T_{n}\right)=\gamma_{n}$. Since $\gamma_{n}$ is $\mathbf{k}\left(\Sigma_{n}\right)$-projective, there is a $\mathbf{k}\left(\Sigma_{n}\right)$-crosssection $s: \gamma_{n}\left(T_{n}\right) \rightarrow \gamma_{n}(B)$. Now the natural transformation

$$
T_{n}(V) \cong \gamma_{n}\left(T_{n}\right) \otimes_{\mathbf{k}\left(\Sigma_{n}\right)} V^{\otimes n} \stackrel{s \otimes \mathrm{id}}{\longrightarrow} \gamma_{n}(B) \otimes_{\mathbf{k}\left(\Sigma_{n}\right)} V^{\otimes n} \stackrel{\Phi_{V}^{B}}{\longrightarrow} B(V)
$$

is a cross-section to $q$ and so $T_{n}$ is projective in $\mathcal{C}$. Since $T_{n} \cong T_{n}^{*}$ is self-dual, $T_{n}$ is also injective in $\mathcal{C}$. The proof is finished.

We remark that if $B$ is a sub-quotient functor of $T_{n}$ with the property that $\gamma_{n}(B)$ is $\mathbf{k}\left(\Sigma_{n}\right)$-projective, it is possible that $B$ is not $T_{n}$-projective. For instance, for the ground field $\mathbf{k}$ being of characteristic 2 , the functor $B=L_{2}^{\text {res }} / L_{2}$ has the property that $\gamma_{2}(B)=0$ with $B$ not $T_{2}$-projective. 


\section{The Structure on Lie Power Functors}

3.1. The Lie Power Functors and the Symmetric Group Modules Lie $(n)$. In this section, the ground ring is a field $\mathbf{k}$. Let $V$ be a module. The free Lie algebra $L(V)$ generated by $V$ is the smallest sub Lie algebra of the tensor algebra $T(V)$ containing $V$, where the Lie structure on $T(V)$ is given by $[a, b]=a b-b a$. The functor $L$ admits a graded structure that

$$
L(V)=\bigoplus_{n=1}^{\infty} L_{n}(V)
$$

where $L_{n}(V)=L(V) \cap T_{n}(V)$ which is called the $n$th Lie power of $V$. By applying equation (2.4) to the functor $L_{n}$, we have the symmetric group module

$$
\operatorname{Lie}(n)=\gamma_{n}\left(L_{n}\right) \text {. }
$$

Let $\bar{V}$ be the $n$-dimensional module with a basis $\left\{x_{1}, \ldots, x_{n}\right\}$ as in subsection 2.3 . From the definition,

$$
\operatorname{Lie}(n)=L_{n}\left(\bar{V}_{n}\right) \cap \gamma_{n}
$$

spanned by the homogenous Lie elements of length $n$ in which each $x_{i}$ occurs exactly once. By the Witt formula, $\operatorname{Lie}(n)$ is of dimension $(n-1)$ !. Following from the antisymmetry and the Jacobi identity, $\operatorname{Lie}(n)$ has a basis given by the elements

$$
\left.\left[\left[x_{1}, x_{\sigma(2)}\right], x_{\sigma(3)}\right], \ldots, x_{\sigma(n)}\right]
$$

for $\sigma \in \Sigma_{n-1}$. (See [5].)

Proposition 3.1. There is a natural short exact sequence

$$
\operatorname{Tor}_{1}^{\mathbf{k}\left(\Sigma_{n}\right)}\left(\gamma_{n} / \operatorname{Lie}(n), V^{\otimes n}\right) \longleftrightarrow \operatorname{Lie}(n) \otimes_{\mathbf{k}\left(\Sigma_{n}\right)} V^{\otimes n} \longrightarrow L_{n}(V)
$$

for any module $V$.

Proof. By Proposition 2.7, it suffices to show that the natural transformation

$$
\Phi_{V}^{L_{n}}: \operatorname{Lie}(n) \otimes_{\mathbf{k}\left(\Sigma_{n}\right)} V^{\otimes n} \longrightarrow L_{n}(V)
$$

is an epimorphism. Let $\left[\left[a_{1}, a_{2}\right], \ldots a_{n}\right] \in L_{n}(V)$ with $a_{1}, \ldots, a_{n} \in V$. Let

$$
\alpha=\left[\left[x_{1}, x_{2}\right], \ldots, x_{n}\right] \in \operatorname{Lie}(n) .
$$

Then exists unique $k_{\sigma} \in \mathbf{k}$ such that

$$
\alpha=\left[\left[x_{1}, x_{2}\right], \ldots, x_{n}\right]=\sum_{\sigma \in \Sigma_{n}} k_{\sigma} x_{\sigma(1)} \cdots x_{\sigma(n)} .
$$

Following the lines in the proof of Proposition 2.7, we have

$$
\Phi_{V}^{L_{n}}\left(\alpha \otimes a_{1} \cdots a_{n}\right)=\sum_{\sigma \in \Sigma_{n}} k_{\sigma} a_{\sigma(1)} \cdots a_{\sigma(n)}=\left[\left[a_{1}, a_{2}\right], \ldots, a_{n}\right] .
$$

The assertion follows from the fact that $L_{n}(V)$ is the $\mathbf{k}$-module spanned by the Lie elements $\left[\left[a_{1}, a_{2}\right], \ldots, a_{n}\right]$ with $a_{j} \in V$.

For any natural transformation $\phi: L_{n} \rightarrow L_{n}$, we have the $\mathbf{k}\left(\Sigma_{n}\right)$-linear map

$$
\gamma_{n}(\phi): \gamma_{n}\left(L_{n}\right)=\operatorname{Lie}(n) \longrightarrow \gamma_{n}\left(L_{n}\right)=\operatorname{Lie}(n) .
$$

This defines a ring homomorphism $\gamma: \operatorname{End}\left(L_{n}\right) \longrightarrow \operatorname{End}_{\mathbf{k}\left(\Sigma_{n}\right)}(\operatorname{Lie}(n))$. 
Proposition 3.2. If $n \neq m$, then $\operatorname{Hom}\left(L_{n}, L_{m}\right)=0$. Moreover the ring homomorphism

$$
\gamma: \operatorname{End}\left(L_{n}\right) \longrightarrow \operatorname{End}_{\mathbf{k}\left(\Sigma_{n}\right)}(\operatorname{Lie}(n))
$$

is an isomorphism with a natural commutative diagram of functors

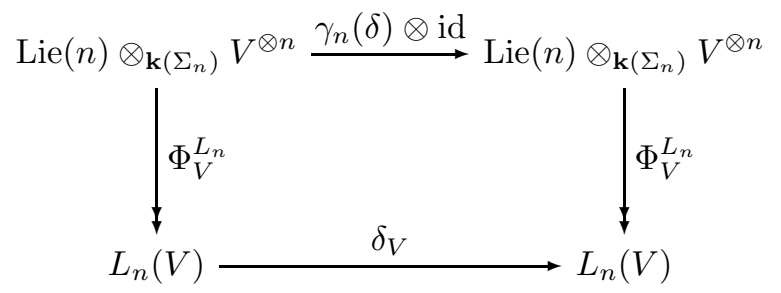

for any natural transformation $\delta: L_{n} \rightarrow L_{n}$.

Proof. Let $\phi: L_{n} \rightarrow L_{m}$ be a natural transformation. Let $\bar{\beta}_{n}: T_{n} \rightarrow L_{n}$ be the natural epimorphism defined by $\bar{\beta}_{n}\left(a_{1} \cdots a_{n}\right)=\left[\left[a_{1}, a_{2}\right], \ldots, a_{n}\right]$ for any module $W$ and any monomial $a_{1} \cdots a_{n} \in T_{n}(W)=W^{\otimes n}$. Then the composite

$$
T_{n} \stackrel{\bar{\beta}}{\longrightarrow} L_{n} \stackrel{\phi}{\longrightarrow} L_{m} \hookrightarrow T_{m}
$$

is a natural transformation, which is zero as $\operatorname{Hom}\left(T_{n}, T_{m}\right)=0$ for $n \neq m$. Thus $\phi=0$.

For the second statement, let $\delta: L_{n} \rightarrow L_{n}$ be a natural transformation. Let $V$ be any module. Consider $\left[\left[a_{1}, a_{2}\right], \ldots, a_{n}\right] \in L_{n}(V)$ with $a_{j} \in V$. Let $f_{a}: \bar{V}_{n} \rightarrow V$ be the linear map with $f_{a}\left(x_{j}\right)=a_{j}$ for $1 \leq j \leq n$. Then there is a commutative diagram

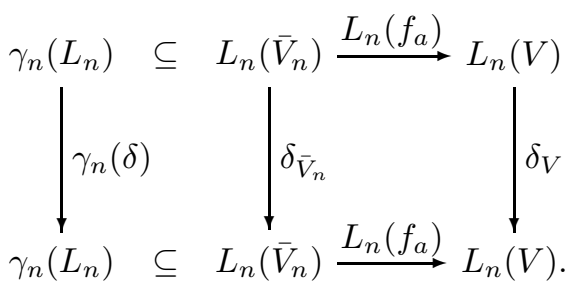

Thus

$$
\begin{array}{rlr} 
& \delta_{V} \circ \Phi_{V}^{L_{n}}\left(\left[\left[x_{1}, x_{2}\right], \ldots, x_{n}\right] \otimes a_{1} \cdots a_{n}\right) & \\
= & \delta_{V}\left(\left[\left[a_{1}, a_{2}\right], \ldots, a_{n}\right]\right) & \text { by equation (3.1) } \\
= & L_{n}\left(f_{a}\right)\left(\gamma_{n}(\delta)\left(\left[\left[x_{1}, x_{2}\right], \ldots, x_{n}\right]\right)\right) & \text { by equation (3.2) } \\
= & \Phi_{V}^{L_{n}}\left(\gamma_{n}(\delta)\left(\left[\left[x_{1}, x_{2}\right], \ldots, x_{n}\right]\right) \otimes a_{1} \cdots a_{n}\right) & \text { by equation (2.9) }
\end{array}
$$

and so the diagram in the statement commutes. It follows that the map

$$
\gamma: \operatorname{End}\left(L_{n}\right) \rightarrow \operatorname{End}_{\mathbf{k}\left(\Sigma_{n}\right)}(\operatorname{Lie}(n))
$$

is a monomorphism.

For showing that $\gamma$ is an epimorphism, let $\theta: \operatorname{Lie}(n) \rightarrow \operatorname{Lie}(n)$ be any $\mathbf{k}\left(\Sigma_{n}\right)$-linear map. Since $\mathbf{k}\left(\Sigma_{n}\right)$ is a Fröbenius algebra, the free $\mathbf{k}\left(\Sigma_{n}\right)$-module $\gamma_{n}$ is injective and 
so there is a commutative diagram of exact sequences of $\mathbf{k}\left(\Sigma_{n}\right)$-modules

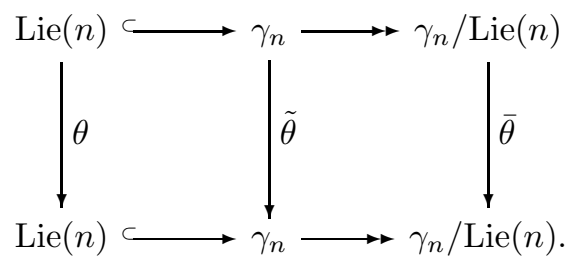

It follows that there is a commutative diagram of short exact sequence of functors

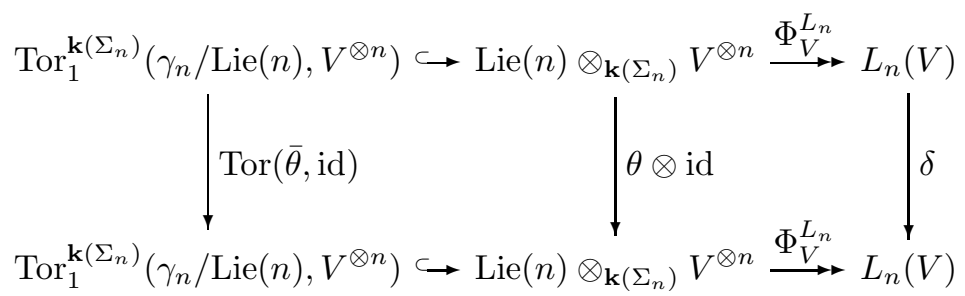

for some natural transformation $\delta: L_{n} \rightarrow L_{n}$. By taking $V=\bar{V}_{n}$ and restricting to the submodule $\gamma_{n} \subseteq \bar{V}^{\otimes n}$, we have the commutative diagram

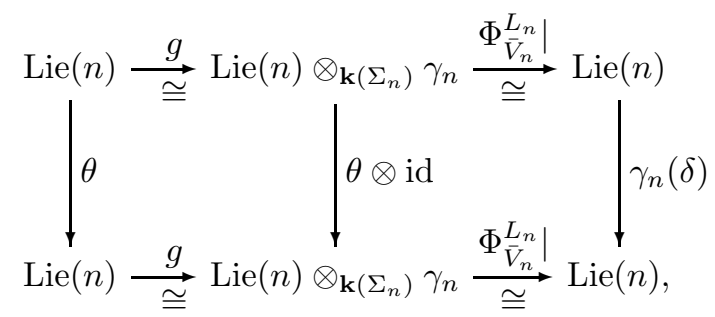

where $g(\alpha)=\alpha \otimes x_{1} \cdots x_{n}$. Thus $\theta=\gamma_{n}(\delta)$ because

$$
\begin{aligned}
\Phi_{\bar{V}}^{L_{n}} \circ g\left(\left[\left[x_{\sigma(1)}, x_{\sigma(2)}\right], \ldots, x_{\sigma(n)}\right]\right) & =\Phi_{\bar{V}}^{L_{n}}\left(\left[\left[x_{1}, x_{2}\right], \ldots, x_{n}\right] \cdot \sigma \otimes x_{1} \cdots x_{n}\right) \\
& =\Phi_{\overline{L_{n}}}^{L_{n}}\left(\left[\left[x_{1}, x_{2}\right], \ldots, x_{n}\right] \otimes \sigma \cdot\left(x_{1} \cdots x_{n}\right)\right) \\
& =\Phi_{\bar{V}}^{L_{n}}\left(\left[\left[x_{1}, x_{2}\right], \ldots, x_{n}\right] \otimes x_{\sigma(1)} \cdots x_{\sigma(n)}\right) \\
& =\left[\left[x_{\sigma(1)}, x_{\sigma(2)}\right], \ldots, x_{\sigma(n)}\right] .
\end{aligned}
$$

The proof is finished.

Corollary 3.3. There is a one-to-one correspondence, multiplicity preserving, between the decompositions of the functor $L_{n}$ and the decompositions of $\operatorname{Lie}(n)$ over $\mathbf{k}\left(\Sigma_{n}\right)$.

3.2. The $T_{n}$-projective Subfunctors of $L_{n}$. Let $Q$ be a subfunctor of $L_{n}$. Then $Q$ is a subfunctor of $T_{n}$ because $L_{n}$ is a subfunctor of $T_{n}$. By Proposition 2.10, the functor $Q$ is $T_{n}$-projective if and only if $\gamma_{n}(Q)$ is a $\mathbf{k}\left(\Sigma_{n}\right)$-projective module. From Corollary 3.3 , we have the following:

Proposition 3.4. There is a one-to-one correspondence, multiplicity preserving, between $T_{n}$-projective sub functors of $L_{n}$ and $\mathbf{k}\left(\Sigma_{n}\right)$-projective submodules of $\operatorname{Lie}(n)$ given by $Q \mapsto \gamma_{n}(Q)$.

According to [22, Lemma 6.2 and Theorem 7.4], there exists a subfunctor $L_{n}^{\max }$ of $L_{n}$ with $\operatorname{Lie}^{\max }(n)=\gamma_{n}\left(L_{n}^{\max }\right)$ that has the following maximum property:

1) $\mathrm{Lie}^{\max }(n)$ is a $\mathbf{k}\left(\Sigma_{n}\right)$-projective submodule of $\operatorname{Lie}(n)$ and 
2) any $\mathbf{k}\left(\Sigma_{n}\right)$-projective submodule of $\operatorname{Lie}(n)$ is isomorphic to a summand of $\mathrm{Lie}^{\max }(n)$ as a $\mathbf{k}\left(\Sigma_{n}\right)$-module.

From the above maximum property, $\operatorname{Lie}^{\max }(n)$ is unique up to isomorphisms of $\mathbf{k}\left(\Sigma_{n}\right)$-modules. By the above proposition, $L_{n}^{\max }$ is unique up to natural equivalences with the maximum property that

1) $L_{n}^{\max }$ is a $T_{n}$-projective subfunctor of $L_{n}$ and

2) any $T_{n}$-projective subfunctor of $L_{n}$ is isomorphic to a summand of $L_{n}^{\max }$.

From Proposition 2.10, we have the following:

Proposition 3.5. There is a natural isomorphism

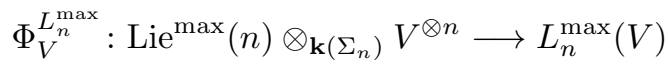

for any module $V$.

3.3. The $\mathbf{k}(\mathrm{GL}(V))$-module $L_{n}(V)$. In this subsection, the ground field $\mathbf{k}$ is an infinite field of characteristic $p>0$ and $V$ is a fixed $\mathbf{k}$-module with the action of the general linear group $\mathrm{GL}(V)=\mathrm{GL}_{m}(\mathbf{k})$ from the right, where $m=\operatorname{dim} V$. Let $\operatorname{GL}(V)$ act on $T_{n}(V)=V^{\otimes n}$ through diagonal, that is,

$$
\left(a_{1} \cdots a_{n}\right) \cdot g=\left(a_{1} g\right) \cdots\left(a_{n} g\right)
$$

for $a_{i} \in V$ and $g \in \mathrm{GL}(V)$. Recall that the Schur algebra is defined by

$$
S(V, n)=\operatorname{End}_{\mathbf{k}\left(\Sigma_{n}\right)}\left(V^{\otimes n}\right),
$$

where the left action of $\Sigma_{n}$ on $V^{\otimes n}$ is given by permuting factors. By the classical Schur-Weyl duality, the group $\operatorname{GL}(V, n)$ generates algebra $S(V, n)=\operatorname{End}_{\mathbf{k}\left(\Sigma_{n}\right)}\left(V^{\otimes n}\right)$ and so there is an epimorphism of rings

$$
\mathbf{k}(\mathrm{GL}(V)) \longrightarrow S(V, n) .
$$

Observe that if $M$ is a sub-quotient of a direct sum of copies of $V^{\otimes n}$, then the $\mathbf{k}(\mathrm{GL}(V))$-action factors through its quotient algebra $S(V, n)$. Thus if $M$ and $N$ are sub-quotients of direct sums of copies of $V^{\otimes n}$, then

$$
\operatorname{Hom}_{\mathbf{k}(\mathrm{GL}(V))}(M, N)=\operatorname{Hom}_{S(V, n)}(M, N) .
$$

Recall from 11] that the category of $\mathbf{k}(\mathrm{GL}(V))$-modules that are sub-quotients of direct sums of copies of $V^{\otimes n}$ is equivalent to the category of modules over the Schur algebra $S(V, n)$, which is denoted by $\operatorname{Mod}(S(V, n))$.

Let $B$ be a sub-quotient of a direct sum of copies of $T_{n}$. The action of $\mathrm{GL}(V)$ on $V$ induces an action on $B(V)$ via the functor $B$. Thus $B(V)$ is a module over $\mathbf{k}(\mathrm{GL}(V))$. Since $B(V)$ is a sub-quotient of a direct sum of copies of $V^{\otimes n}, B(V)$ is an object in $\operatorname{Mod}(S(V, n))$. Thus we have a functor:

$$
\begin{array}{ll}
\Theta: \quad & B \mapsto B(V) \\
& \operatorname{Hom}(A, B) \longrightarrow \operatorname{Hom}_{\mathbf{k}(\mathrm{GL}(V))}(A(V), B(V))=\operatorname{Hom}_{S(V, n)}(A(V), B(V))
\end{array}
$$

from the category of sub-quotients of direct sums of copies of $T_{n}$ to $\operatorname{Mod}(S(V, n))$.

Lemma 3.6. Let $B$ be a sub-quotient of a free $T_{n}$-functor and let $V$ be a module with $\operatorname{dim}(V) \geq n$. Then $B=0$ if and only if $B(V)=0$. 
Proof. If $B=0$, clearly $B(V)=0$. Assume that $B(V)=0$. Let $B=\tilde{B} / B^{\prime}$ with $B^{\prime} \hookrightarrow \tilde{B} \hookrightarrow F$, where $F$ is a direct sum of copies of $T_{n}$. It is routine to check that $\gamma_{j}\left(T_{n}\right)=0$ for $j>n$. Thus $\gamma_{j}(F)=0$ for $j>n$ and so

$$
\gamma_{j}\left(B^{\prime}\right)=\gamma_{j}(\tilde{B})=\gamma_{j}(B)=0
$$

for $j>n$. Since $B(V)=0$, we have $B\left(\bar{V}_{n}\right)=0$ because $\operatorname{dim} V \geq \operatorname{dim} \bar{V}_{n}=n$. Thus $\gamma_{j}(B)=0$ for $j \leq n$. The assertion follows by Corollary 2.6.

Corollary 3.7. Let $A$ and $B$ sub-quotients of free $T_{n}$-functors and let $V$ be a module with $\operatorname{dim}(V) \geq n$. Then

$$
\Theta: \operatorname{Hom}(A, B) \longrightarrow \operatorname{Hom}_{\mathbf{k}(\mathrm{GL}(V))}(A(V), B(V))=\operatorname{Hom}_{S(V, n)}(A(V), B(V))
$$

is a monomorphism.

Proof. Let $f: A \rightarrow B$ be a natural transformation such that $f_{V}: A(V) \rightarrow B(V)$ is 0. Let $C=\operatorname{Im}(f: A \rightarrow B)$. Then $C(V)=0$. Thus $C=0$ and hence the result.

A direct sum of finite copies of $T_{n}$ is called a finite free $T_{n}$-functor.

Proposition 3.8. Let $B$ be a sub-quotient of a finite free $T_{n}$-functor and let $A$ be a quotient functor of a finite free $T_{n}$-functor. Suppose that $\operatorname{dim} V \geq n$. Then the homomorphism

$$
\Theta_{A, B}: \operatorname{Hom}(A, B) \longrightarrow \operatorname{Hom}_{\mathbf{k}(\mathrm{GL}(V))}(A(V), B(V))=\operatorname{Hom}_{S(V, n)}(A(V), B(V))
$$

is an isomorphism.

Proof. By Schur-Weyl duality, the monomorphism

$$
\Theta_{T_{n}, T_{n}}: \operatorname{Hom}\left(T_{n}, T_{n}\right) \longrightarrow \operatorname{Hom}_{\mathbf{k}(\mathrm{GL}(V))}\left(T_{n}(V), T_{n}(V)\right)
$$

is an epimorphism and so $\Theta_{A, B}$ is an isomorphism when $A$ and $B$ are free $T_{n^{-}}$ functors. According to [8, p.94], $V^{\otimes n}$ is projective over $S(V, n)$. Let $A$ be a free $T_{n}$ functor. By tracking the exact sequence for

$$
\Theta_{A,-}: \operatorname{Hom}(A,-) \longrightarrow \operatorname{Hom}_{S(V, n)}(A(V),-)
$$

together with the fact that $\Theta_{A, B}$ is always a monomorphism, we have

$$
\Theta_{A, B}: \operatorname{Hom}(A, B) \longrightarrow \operatorname{Hom}_{S(V, n)}(A(V), B(V))
$$

for any sub-quotient $B$ of a free $T_{n}$-functor. Let $A$ be a quotient of a free functor $F$ with an epimorphism $\phi: F \rightarrow A$. Let $C=\operatorname{Ker}(\phi)$. From the commutative diagram of exact sequences

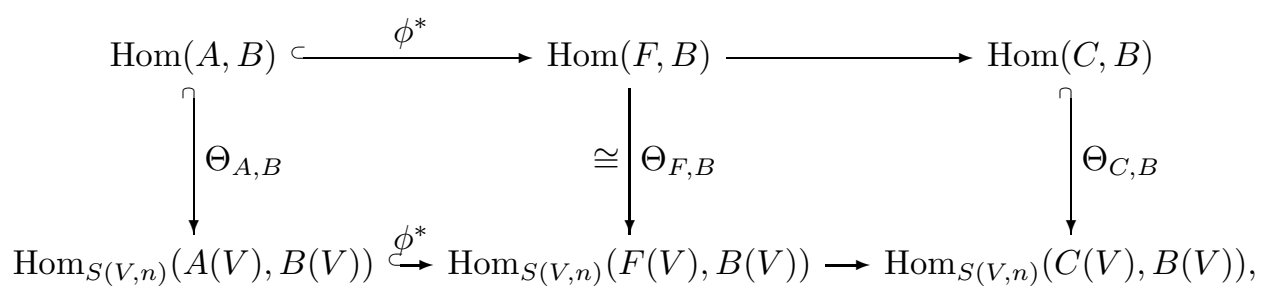

the monomorphism $\Theta_{A, B}$ is an epimorphism and hence the result. 
A $\mathbf{k}(\mathrm{GL}(V))$-submodule $M$ of $L_{n}(V)$ is called $T_{n}$-projective if $M$ is isomorphic to a summand of a direct summation of $T_{n}(V)$ as modules over $\mathbf{k}(\mathrm{GL}(V))$. Let $\bar{\beta}_{n}: T_{n} \rightarrow L_{n}$ be the natural epimorphism defined by $\bar{\beta}_{n}\left(a_{1} \cdots a_{n}\right)=\left[\left[a_{1}, a_{2}\right], \ldots, a_{n}\right]$ for any module $W$ and any monomial $a_{1} \cdots a_{n} \in T_{n}(W)=W^{\otimes n}$. Let $\beta_{n}$ be the composite $T_{n} \stackrel{\bar{\beta}_{n}}{\longrightarrow} L_{n} \stackrel{i}{\longrightarrow} T_{n}$.

Theorem 3.9. Suppose that $\operatorname{dim} V \geq n$. Then

(1). The ring homomorphism

$$
\Theta_{L_{n}, L_{n}}: \operatorname{Hom}\left(L_{n}, L_{n}\right) \longrightarrow \operatorname{Hom}_{\mathbf{k}(\mathrm{GL}(V))}\left(L_{n}(V), L_{n}(V)\right)
$$

is an isomorphism.

(2). There is a one-to-one correspondence, multiplicity preserving between summands of the functor $L_{n}$ and $\mathbf{k}(\mathrm{GL}(V))$-summands of $L_{n}(V)$.

(3). There is a one-to-one correspondence, multiplicity preserving between $T_{n}$ projective subfunctors of $L_{n}$ and $T_{n}$-projective $\mathbf{k}(\mathrm{GL}(V))$-submodules of $L_{n}(V)$.

(4). For the functor $L_{n}^{\max }$, the module $L_{n}^{\max }(V)$ is the maximum $T_{n}$-projective submodule of $L_{n}(V)$ in the sense that any $T_{n}$-projective $\mathbf{k}(\mathrm{GL}(V))$-submodule of $L_{n}(V)$ is isomorphic to a summand of $L_{n}^{\max }(V)$.

(5). For any choice of the functor $L_{n}^{\max }$, the socle $\operatorname{Soc}\left(L_{n}^{\max }(V)\right)$ is uniquely determined by

$$
\bar{\beta}_{n}\left(\operatorname{Soc}\left(V^{\otimes n}\right)\right)=\beta_{n}\left(\operatorname{Soc}\left(V^{\otimes n}\right)\right) .
$$

as the submodule of $L_{n}(V) \subseteq T_{n}(V)$.

(6). For any choice of the functor $L_{n}^{\max }$, the head $\operatorname{Hd}\left(L_{n}^{\max }(V)\right)$ is uniquely determined by

$$
\beta_{n}\left(\operatorname{Hd}\left(V^{\otimes n}\right)\right) .
$$

as the quotient module of $T_{n}(V)$.

Note. By assertion (5), the functor $L_{n}^{\max }$ and the module $L_{n}^{\max }(V)$ are determined by evaluating the map $\bar{\beta}_{n}$ or $\beta_{n}$ on simple $\mathbf{k}(\operatorname{GL}(V))$-submodules of $V^{\otimes n}$.

Proof. Since $L_{n}$ is a quotient functor of $T_{n}$, assertion (1) is direct consequence of Proposition 3.8. Assertion (2) follows from (1) immediately. Assertion (4) is a direct consequence of 3 . The proof of assertion (6) is similar to that of assertion (5).

For proving assertion (3), let $M$ be a $T_{n}$-projective $\mathbf{k}(\mathrm{GL}(V))$-submodule of $L_{n}(V)$. According to [8, p.94], $T_{n}(V)$ is an injective module over $S(V, n)$ and so is $M$ because $M$ is a summand of a direct sum of finite copies of $T_{n}$. Thus the inclusion

$$
j: M \hookrightarrow L_{n}(V) \hookrightarrow T_{n}(V)
$$

admits a $\mathbf{k}(\mathrm{GL}(V))$-retraction $r: T_{n}(V) \rightarrow M$. The composite

$$
e=j \circ r: T_{n}(V) \rightarrow T_{n}(V)
$$

is an idempotent in

$$
\operatorname{End}_{\mathbf{k}(\mathrm{GL}(V))}\left(T_{n}(V)\right) \text {. }
$$

The natural transformation $\alpha=\Theta^{e}: T_{n} \rightarrow T_{n}$ is an idempotent. Let $B=\operatorname{Im}(\alpha)$. Then $B$ is a $T_{n}$-projective subfunctor of $L_{n}$ with $B(V)=M$ and hence assertion (3). 
(5). Let

$$
V^{\otimes n}=\bigoplus_{i \in I} P_{i}
$$

be a decomposition over $S(V, n)$ such that each $P_{i}$ is indecomposable. The map $\bar{\beta}_{n}: V^{\otimes n} \rightarrow L_{n}(V)$ induces a map

$$
\bar{\beta}_{n}: \operatorname{Soc}\left(V^{\otimes n}=\bigoplus_{i \in I} \operatorname{Soc}\left(P_{i}\right) \longrightarrow \operatorname{Soc}\left(L_{n}(V)\right) .\right.
$$

Note that each indecomposable $S(V, n)$-summand of $V^{\otimes n}$ has a unique socle. (See for instance [11, (6.4b)]. Thus there exists $I^{\prime} \subseteq I$ such that

$$
P=\bigoplus_{i \in I^{\prime}} P_{i}
$$

has the property that

$$
\bar{\beta}_{n} \mid: \operatorname{Soc}(P) \longrightarrow \bar{\beta}_{n}\left(\operatorname{Soc}\left(V^{\otimes n}\right)\right)
$$

is an isomorphism. It follows that

$$
\bar{\beta}_{n} \mid: P \longrightarrow L_{n}(V)
$$

is a monomorphism because it restricts to the socle is a monomorphism. Since $P$ is an injective $S(V, n)$-module, the map $\left.\bar{\beta}_{n}\right|_{P}$ has a retraction. Thus $T_{n}$-projective $S(V, n)$-module $P$ is isomorphic to a $S(V, n)$-summand of $L_{n}(V)$. From the maximum property of $L^{\max }(n), P$ is isomorphic to a $S(V, n)$-summand of $L_{n}^{\max }(V)$. In particular,

$$
\bar{\beta}_{n}\left(\operatorname{Soc}\left(V^{\otimes n}\right)\right)=\bar{\beta}_{n} \mid(\operatorname{Soc}(P)) \subseteq \operatorname{Soc}\left(L_{n}^{\max }(V)\right) .
$$

On the other hand, since $L_{n}^{\max }(V)$ is $S(V, n)$-projective, the inclusion

$$
j: L_{n}^{\max }(V) \hookrightarrow L_{n}(V)
$$

admits a $S(V, n)$-lifting $\tilde{j}: L_{n}^{\max }(V) \rightarrow V^{\otimes n}$ such that $j=\bar{\beta} \circ \tilde{j}$. Thus

$$
\operatorname{Soc}\left(L_{n}^{\max }(V)\right) \subseteq \bar{\beta}_{n}\left(\operatorname{Soc}\left(V^{\otimes n}\right)\right) .
$$

Note that the inclusion $i: L_{n}(V) \hookrightarrow T_{n}(V)$ induces a monomorphism $i \mid: \operatorname{Soc}\left(L_{n}(V)\right) \hookrightarrow$ $\operatorname{Soc}\left(T_{n}(V)\right)$. Thus

$$
\bar{\beta}_{n}\left(\operatorname{Soc}\left(V^{\otimes n}\right)\right)=\beta_{n}\left(\operatorname{Soc}\left(V^{\otimes n}\right)\right)
$$

and hence the result.

We give a remark that if $\operatorname{dim} V<n$, then assertions (3) and (4) are not true by the following example.

Example 3.10. Let $\mathbf{k}$ be of characteristic 3 and let $V$ be a 2-dimensional module with a basis $\{u, v\}$. Then the canonical map

$$
f: L_{2}(V) \otimes V \longrightarrow L_{3}(V)\left[a_{1}, a_{2}\right] \otimes a_{3} \mapsto\left[\left[a_{1}, a_{2}\right], a_{3}\right]
$$

is an isomorphism of modules over $\mathbf{k}\left(\mathrm{GL}_{2}(\mathbf{k})\right)$. Since $L_{2}(V)$ is a $\mathbf{k}(\mathrm{GL}(V))$-summand of $V^{\otimes 2}, L_{2}(V) \otimes V$ is $T_{3}$-projective. Thus $L_{3}(V)$ is $T_{3}$-projective. On the other hand, it is easy to see that the functor $L_{3}^{\max }=0$ and so $L_{3}^{\max }(V)=0$.

In this case, $L_{3}(V)$ is not an injective $S(V, 3)$-module. In fact, the inclusion $L_{3}(V) \hookrightarrow V^{\otimes 3}$ does not have $S(V, 3)$-retraction by expecting the Steenrod module structure on $V^{\otimes 3}$. Also it is easy to check that $L_{3}(V)$ is not a projective $S(V, 3)$ module. 
We call $M \subseteq L_{n}(V)$ functorial $T_{n}$-projective if there exists a $T_{n}$-projective subfunctor $Q$ of $L_{n}$ such that $M=Q(V)$. (Note. Here we require that $Q(V)$ is strictly equal to $M$ rather than isomorphic to $M$.)

Proposition 3.11. Assume that the ground field $\mathbf{k}$ has infinite elements. Let $V$ be any $\mathbf{k}$-module and let $M$ be $a \mathbf{k}(\mathrm{GL}(V))$-submodule of $L_{n}(V)$. Then $M$ is functorial $T_{n}$-projective if and only if $M$ satisfies the following two conditions:

1) There exists a $\mathbf{k}(\mathrm{GL}(V))$-linear map $r: V^{\otimes n} \rightarrow M$ such that $\left.r\right|_{M}$ is the identity.

2) The inclusion $M \hookrightarrow L_{n}(V)$ admits the following lifting

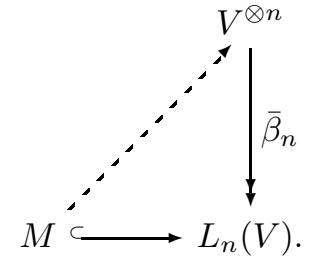

as modules over $\mathbf{k}(\mathrm{GL}(V))$.

Proof. Suppose that $M$ is functorial $T_{n}$-projective. Let $Q$ be a subfunctor of $L_{n}$ with $Q(V)=M$. The inclusion

$$
Q \hookrightarrow L_{n} \longrightarrow T_{n}
$$

admits a natural retraction because $\gamma_{n}(Q)$ is injective. By evaluating at $V$, condition 1 is satisfied. Since $\gamma_{n}(Q)$ is projective, there a natural lifting $\tilde{j}: Q \rightarrow T_{n}$ such that $\beta_{n} \circ \tilde{j}$ is the inclusion of $Q$ in $L_{n}$ and so condition 2 is satisfied by evaluating at $V$.

Conversely suppose that $M$ satisfies conditions 1 and 2 . Let $j: M \hookrightarrow L_{n}(V)$ and $L_{n}(V) \hookrightarrow V^{\otimes n}$ be the inclusions. Let $\tilde{j}: M \rightarrow V^{\otimes n}$ be a $\mathbf{k}(\mathrm{GL}(V))$-map such that $\beta_{n} \circ \tilde{j}=j$. By the Schur-Weyl duality, the map

$$
\mathbf{k}\left(\Sigma_{n}\right)=\operatorname{Hom}\left(T_{n}, T_{n}\right) \longrightarrow \operatorname{End}_{\mathbf{k}(\mathrm{GL}(V))}\left(V^{\otimes n}\right)
$$

is an epimorphism. There exists a natural transformation $\alpha: T_{n} \rightarrow T_{n}$ such that $\alpha_{V}=\tilde{j} \circ r$. Let $\theta=\beta_{n} \circ \alpha$. Consider the colimit of the sequence

$$
T_{n} \stackrel{\theta}{\longrightarrow} T_{n} \stackrel{\theta}{\longrightarrow} T_{n} \longrightarrow \cdots,
$$

there exists $k>>0$ such that

$$
Q=\operatorname{Im}\left(\theta^{k}\right) \longrightarrow \operatorname{colim}_{\theta} T_{n}
$$

is an isomorphism because, by taking $\gamma_{n}(-)$ to the above sequence, the submodules $\operatorname{Im}\left(\gamma_{n}\left(\theta^{t}\right)\right)$ of $\gamma_{n}\left(T_{n}\right)$ has the monotone decreasing in dimensions:

$$
\operatorname{dim} \operatorname{Im}\left(\gamma_{n}(\theta)\right) \geq \operatorname{dim} \operatorname{Im}\left(\gamma_{n}\left(\theta^{2}\right)\right) \geq \operatorname{dim} \operatorname{Im}\left(\gamma_{n}\left(\theta^{3}\right)\right) \geq \cdots .
$$

Since $Q=\beta_{n}\left(\alpha \theta^{k-1}\left(T_{n}\right)\right), Q$ is a $T_{n}$-projective subfunctor of $L_{n}$. By evaluating at $V$, we check that $Q(V)=M$. Since

$$
\begin{aligned}
\theta_{V} \circ \theta_{V} & =\beta_{n} \circ \alpha_{V} \circ \beta_{n} \circ \alpha_{V} \\
& =i \circ \bar{\beta}_{n} \circ \tilde{j} \circ r \circ i \circ \bar{\beta}_{n} \circ \tilde{j} \circ r \\
& =i \circ j \circ r \circ i \circ j \circ r \\
& =i \circ j \circ r \\
& =\theta_{V},
\end{aligned}
$$


the map $\theta_{V}$ is an idempotent. It follows that

$$
Q(V)=\operatorname{Im}\left(\theta_{V}\right)=i \circ j \circ r\left(V^{\otimes n}\right)=M
$$

and hence the result.

\section{Coalgebra Structure on Tensor Algebras}

In this section, the tensor algebra $T(V)$ admits the comultiplication

$$
\psi: T(V) \longrightarrow T(V) \otimes T(V)
$$

described in subsection 2.1

4.1. Changing Ground-rings. Some results in the representation theory help us to change the ground ring. Let $\mathbb{Z}_{(p)}$ be the $p$-local integers. By modular representation theory of symmetric groups (see, for example [7, Exercise 6.16, p.142]), any idempotent in $(\mathbb{Z} / p)\left(\Sigma_{n}\right)$ lifts to an idempotent in $\mathbb{Z}_{(p)}\left(\Sigma_{n}\right)$. It is well-known [14] that any irreducible modules $M$ over $\mathbb{Z} / p\left(\Sigma_{n}\right)$ is absolutely irreducible, that is, for any extension field $\mathbf{k}, M \otimes \mathbf{k}$ is irreducible over $\mathbf{k}\left(\Sigma_{n}\right)$. Thus there is a one-to-one correspondence between idempotents in $\mathbb{Z} / p\left(\Sigma_{n}\right)$ and the idempotents in $\mathbf{k}\left(\Sigma_{n}\right)$.

Let $R$ be any commutative ring with identity. Consider $T: V \mapsto T(V)$ as the functor from projective $R$-modules to coalgebras over $R$. Denote by $\operatorname{coalg}^{R}(T, T)$ the set of self natural coalgebra transformation of $T$. Let $\mathbf{k}$ be any field of characteristic $p$. We have the canonical functions

$$
R: \operatorname{coalg}^{\mathbb{Z}(p)}(T, T) \longrightarrow \operatorname{coalg}^{\mathbb{Z} / p}(T, T)
$$

by modulo $p$ and

$$
K: \operatorname{coalg}^{\mathbb{Z} / p}(T, T) \longrightarrow \operatorname{coalg}^{\mathbf{k}}(T, T)
$$

by tensoring with $\mathbf{k}$ over $\mathbb{Z} / p$. By [22, Corollary 6.9], there is a one-to-one correspondence between natural indecomposable retract of $T$ over $\mathbf{k}$ and the indecomposable $\mathbf{k}\left(\Sigma_{n}\right)$-projective submodule of $\operatorname{Lie}(n)$ for $n \geq 1$. Thus we have the following:

Proposition 4.1. The functions $R$ and $K$ have the following properties:

(1). The map $R$ : coalg ${ }^{\mathbb{Z}(p)}(T, T) \longrightarrow \operatorname{coalg}^{\mathbb{Z} / p}(T, T)$ induces a one-to-one correspondence betweens idempotents. Thus every natural coalgebra decompositions of $T$ over $\mathbb{Z} / p$ lifts to a natural coalgebra decomposition over $\mathbb{Z}_{(p)}$.

(2). The map $K: \operatorname{coalg}^{\mathbb{Z}_{p}}(T, T) \longrightarrow \operatorname{coalg}^{\mathbf{k}}(T, T)$ induces a one-to-one correspondence betweens idempotents. Thus natural coalgebra decompositions of $T$ only depends on the characteristic of the ground field.

By this proposition, we can freely change the ground fields $\mathbf{k}$ with the same characteristic and lift natural coalgebra decompositions over $p$-local integers if it is necessary.

4.2. Block Decompositions. From now on in this section, the ground field $\mathbf{k}$ is algebraically closed with $\operatorname{char}(\mathbf{k})=p$. For any coalgebra $C$, let $P C$ be the set of the primitives of $C$. If $C$ is a functor from modules to coalgebras, then $P C$ is a functor from modules to modules. Recall from Proposition 2.3 that any subfunctor of $T$ is a graded subfunctor. Thus if $C$ is sub-quotient coalgebra functor of $T^{C}$, then $C$ is graded and so we have the homogenous functors $C_{n}$ and $P_{n} C=P C \cap C_{n}$ for each $n$. For the case $C=T, P T(V)=L^{\text {res }}(V)$ is the free restricted Lie algebra generated by $V$ and $P_{n} T=L_{n}^{\text {res }}$ for each $n$. 
For natural transformations $f, g: T \rightarrow T$, the convolution product $f * g$ is defined by the composite

$$
T(V) \stackrel{\psi}{\longrightarrow} T(V) \otimes T(V) \stackrel{f \otimes g}{\longrightarrow} T(V) \otimes T(V) \stackrel{\text { multi. }}{\longrightarrow} T(V) .
$$

If $f$ and $g$ are natural coalgebra transformation, clearly $f * g$ is also a natural coalgebra transformation. For any element $\zeta \in \mathbf{k}$, define $\lambda_{\zeta}: T(V) \rightarrow T(V)$ by setting

$$
\lambda_{\zeta}\left(a_{1} \cdots a_{n}\right)=\zeta^{n} a_{1} \cdots a_{n}
$$

for $a_{1}, \ldots, a_{n} \in V$. In other words, $\lambda_{\zeta}: T(V) \rightarrow T(V)$ is the (unique) Hopf map such that $\lambda_{\zeta}(a)=\zeta a$ for $a \in V$. Let $\chi: T(V) \rightarrow T(V)$ be the conjugation of the Hopf algebra $T(V)$, namely $\chi$ is the anti-homomorphism such that $\chi(a)=-a$ for $a \in V$. More precisely,

$$
\chi\left(a_{1} \cdots a_{n}\right)=(-1)^{n} a_{n} a_{n-1} \cdots a_{1}
$$

for $a_{1}, \ldots, a_{n} \in V$. For any element $\zeta \in \mathbf{k}$, we have the natural coalgebra transformation

$$
\theta_{\zeta}=\lambda_{\zeta} * \chi: T(V) \rightarrow T(V)
$$

If $\alpha \in P_{n} T(V)$, then

$$
\theta_{\zeta}(\alpha)=\left(\zeta^{n}-1\right) \alpha
$$

by the definition of convolution product because $\psi(\alpha)=\alpha \otimes 1+1 \otimes \alpha$. For general monomials in $T_{n}(V)$, it is straightforward to have the formula

$$
\begin{aligned}
& \theta_{\zeta}\left(a_{1} \cdots a_{n}\right)=\sum_{\sigma(1)<\cdots<\sigma(k)} \\
& \sigma(k+1)<\cdots<\sigma(n) \\
& \sigma \in \Sigma_{n} \\
& 0 \leq k \leq n \\
& \left(\zeta^{k}+(-1)^{n-k}\right) a_{\sigma(1)} \cdots a_{\sigma(k)} a_{\sigma(k+1)} \cdots a_{\sigma(n)} .
\end{aligned}
$$

The maps $\theta_{\zeta}$ are useful for obtaining natural coalgebra decompositions of $T(V)$.

Theorem 4.2. Let the ground ring $\mathbf{k}$ be a field of characteristic $p$. Then there exists a natural coalgebra decomposition

$$
T(V) \cong C(V) \otimes D(V)
$$

for any module $V$ with the property that

$$
P C_{n}= \begin{cases}0 & \text { if } n \text { is not a power of } p, \\ P_{n} T & \text { if } \quad n=p^{r} \text { for some } r .\end{cases}
$$

Note. From the decomposition, we have $P_{n} D=0$ if $n$ is a power of $p$ and $P_{n} D=P_{n} T$ if $n$ is not a power of $p$. The theorem gives a decomposition that one can put all primitives of tensor length of powers of $p$ in a one coalgebra factor and put the rest primitives in another coalgebra factor.

Proof. Let $\left\{m_{1}<m_{2}<m_{3}<\cdots\right\}$ be the set of all positive integers prime to $p$ excluding 1 and let $\zeta_{m_{i}}$ be a primitive $m_{i}$ th root of 1 . We are going to construct by induction a sequence of sub coalgebra functor $C(k)$ of $T$, with the inclusion denoted by $j_{k}: C(k) \hookrightarrow T$, and a sequence of quotient coalgebra functor $q_{k}: T \rightarrow E(k)$ with the following properties: 
1) $C(k+1)$ is a subfunctor $C(k)$ for each $k \geq 0$.

2) There exists a coalgebra natural transformation $q_{k}^{\prime}: E(k) \rightarrow E(k+1)$ such that $q_{k+1}=q_{k}^{\prime} \circ q_{k}$ for each $k \geq 0$.

3) The composite $q_{k} \circ j_{k}: C(k) \rightarrow E(k)$ is a natural isomorphism.

4) The primitives $P_{n} C(k)=0$ if $n$ is divisible by one of $m_{1}, m_{2}, \ldots, m_{k}$.

5) The primitive $P_{n} C(k)=P_{n} T$ if $n$ is not divisible by any of $m_{1}, m_{2}, \ldots, m_{k}$. Let $C(0)=E(0)=T$ and let $i_{0}=q_{0}=\mathrm{id}$. The construction of $C(1)$ and $E(1)$ is as follows. Let $E(1)=\operatorname{colim}_{\theta_{\zeta_{m_{1}}}} T$ be the colimit of the sequence of coalgebra natural transformation

$$
T \stackrel{\theta_{\zeta m_{1}}}{\longrightarrow} T \stackrel{\theta_{\zeta m_{1}}}{\longrightarrow} T \longrightarrow \cdots .
$$

Let $q_{1}: T \rightarrow E(1)$ be the map to its colimit. By [22, Theorem 4.5], there exists a sub coalgebra functor $C(1)$ of $T$, with the inclusion denoted by $j_{1}: C(1) \rightarrow T$, such that $q_{1} \circ i_{1}$ is a natural isomorphism. From Equation 4.4 .

$$
\theta_{\zeta_{m_{1}}}: P_{n} T \rightarrow P_{n} T
$$

is zero if $m_{1} \mid n$ and an isomorphism if $m_{1} \nmid n$. Thus $P_{n} E(1)=\operatorname{colim}_{\theta_{\zeta_{m_{1}}}} P_{n} T=0$ if $m_{1} \mid n$ and

$$
q_{1}: P_{n} T \longrightarrow P_{n} E(1)
$$

is an isomorphism if $m_{1} \nmid n$. Since $C(1) \cong E(1)$, conditions (4) and (5) holds. Now suppose that we have construct $C(j)$ and $E(j)$ satisfying conditions (1)-(5) for $j \leq k$. Let $f: T \rightarrow T$ be the composite

$$
T \stackrel{q_{k}}{\longrightarrow} E_{n} \stackrel{\left(q_{k} \circ j_{k}\right)^{-1}}{\cong} C(k) \stackrel{j_{k}}{\longrightarrow} T \stackrel{\theta_{\zeta_{m_{k+1}}}}{\longrightarrow} T
$$

and let $E(k+1)=\operatorname{colim}_{f} T$. Let $q_{k+1}: T \rightarrow E(k+1)$ be the canonical map to its colimit. Notice that

$$
q_{k+1} \circ f=q_{k+1}: T \longrightarrow E(k+1) .
$$

Let $q_{k}^{\prime}=q_{k+1} \circ j_{k} \circ\left(q_{k} \circ j_{k}\right)^{-1}$. Then $q_{k+1}=q_{k}^{\prime} \circ q_{k}$ and so condition (2) satisfies. Since $f$ factors through the subfunctor $C(k)$, there exists a subfunctor $C(k+1)$ of $C(k)$, with the inclusion into $T$ denoted by $j_{k+1}$, such that $q_{k+1} \circ j_{k+1}$ is a natural isomorphism. Hence we have conditions (1) and (3). Let $\alpha \in P_{n} T(V)$. Then

$$
f(\alpha)=\theta_{\zeta_{m_{k+1}}}\left(\left(j_{k} \circ\left(q_{k} \circ j_{k}\right)^{-1} \circ q_{k}\right)(\alpha)\right)=\left(\zeta_{m_{k+1}}^{n}-1\right)\left(\left(j_{k} \circ\left(q_{k} \circ j_{k}\right)^{-1} \circ q_{k}\right)(\alpha)\right. \text {. }
$$

Thus $f(\alpha)=0$ if $n$ is divisible by one of $m_{1}, \ldots, m_{k+1}$ and

$$
f: P_{n} T \longrightarrow P_{n} T
$$

is an isomorphism if $n$ is not divisible by any of $m_{1}, \ldots, m_{k+1}$. It follows that $P_{n} E(k+1)=0$ if $n$ is divisible by one of $m_{1}, \ldots, m_{k+1}$ and

$$
q_{k+1}: P_{n} T \longrightarrow P_{n} E(k+1)
$$

is an isomorphism if $n$ is not divisible by any of $m_{1}, \ldots, m_{k+1}$. Since $C(k+1) \cong$ $E(k+1)$, we have conditions (4) and (5). The induction is finished.

Now let

$$
C=\bigcap_{k=0}^{\infty} C(k)
$$

be the intersection of the subfunctors $C(k)$ of $T$ and let $E(\infty)$ be the colimit of the sequence

$$
T \stackrel{q_{1}}{\longrightarrow} E(1) \stackrel{q_{2}^{\prime}}{\longrightarrow} E(2) \stackrel{q_{3}^{\prime}}{\longrightarrow} E(3) \longrightarrow \cdots
$$


From condition (3), each $C(k)$ is coalgebra retract of $T$ and so each $C(k)$ is a functor from modules to coassociative and cocommutative quasi-Hopf algebras with the multiplication on $C(k)$ given by

$$
C(k) \otimes C(k) \subset T \otimes T \stackrel{\text { multi }}{\longrightarrow} T \longrightarrow C(k),
$$

where we use the notation of quasi-Hopf algebra given in [18. By conditions (1)(3), $C(k+1)$ is a coalgebra retract of $C(k)$ and so there is a natural coalgebra decomposition

$$
C(k) \cong C(k+1) \otimes C^{\prime}(k)
$$

by [22, Lemma 5.3]. From conditions (4) and (5), $P_{n} C(k+1)=P_{n} C(k)$ for $n<m_{k+1}$ and so $P_{n} C^{\prime}(k)=0$ for $n<m_{k+1}$. It follows that

$$
C^{\prime}(k)_{n}=0
$$

for $0<n<m_{k+1}$. Thus

$$
C(k+1)_{n}=C(k)_{n}
$$

for $n<m_{k+1}$ and from conditions (1)-(3),

$$
q_{k}: E(k)_{n} \longrightarrow E(k+1)_{n}
$$

is an isomorphism for $n<m_{k+1}$. Notice that the integers $m_{k} \rightarrow \infty$ as $k \rightarrow \infty$. Let $n$ be a fixed positive integer. For the integers $k$ with $m_{k}>n$, we have $C_{n}=C(k)_{n}$ and

$$
E(k)_{n} \underset{q_{k}^{\prime}}{\stackrel{\longrightarrow}{\longrightarrow}} E(k+1)_{n} \underset{q_{k+1}^{\prime}}{\cong} E(k+2)_{n} \cdots \cdots
$$

It follows that the composite

$$
C_{n}=C(k)_{n} \stackrel{j_{k}}{\longrightarrow} T_{n} \stackrel{q_{k}}{\longrightarrow} E(k)_{n} \longrightarrow E(\infty)_{n}
$$

is an isomorphism. Thus the composite

$$
C \longrightarrow T \longrightarrow E(\infty)
$$

is an isomorphism and so $C$ is a coalgebra retract of $T$. This gives a natural coalgebra decomposition

$$
T \cong C \otimes D
$$

for some coalgebra retract $D$ of $T$. From conditions (4) and (5), we have $P_{n} C=0$ if $n$ is not a power of $p$ and $P C_{p^{r}}=P T_{p^{r}}$ for $r \geq 0$. The proof is finished.

Theorem 4.3 (Block Decomposition Theorem). Let $\mathbf{k}$ be a field of characteristic p. Let $\left\{m_{i}\right\}_{i \geq 0}$ be the set of all positive integers prime to $p$ with the order that $m_{0}=1<m_{1}<m_{2}<\cdots$. Then there exist natural coalgebra retracts $C^{m_{i}}$ of $T$ with a natural coalgebra decomposition

$$
T(V) \cong \bigotimes_{i=0}^{\infty} C^{m_{i}}(V)
$$

such that

$$
P_{n} C^{m_{i}}=\left\{\begin{array}{ccc}
P_{n} T & \text { if } & n=m_{i} p^{r} \text { for some } r \geq 0 \\
0 & \text { otherwise }
\end{array}\right.
$$


Proof. We are going to show by induction that there exists natural coalgebra retracts $C^{m_{i}}$ of $T$, for $0 \leq i \leq k$, with a natural coalgebra decomposition

$$
T(V) \cong\left(\bigotimes_{i=0}^{k} C^{m_{i}}(V)\right) \otimes D^{k}(V)
$$

for some natural coalgebra retract $D^{k}$ of $T$ such that

$$
P_{n} C^{m_{i}}=\left\{\begin{array}{ccc}
P_{n} T & \text { if } & n=m_{i} p^{r} \text { for some } r \geq 0, \\
0 & \text { otherwise }
\end{array}\right.
$$

for $0 \leq i \leq k$. The statement holds for $k=0$ by Theorem 4.2 where $C^{m_{0}}$ is the natural coalgebra retract $C$ of $T$ given in Theorem 4.2. Suppose that the statement holds for $k$. Following the lines in the proof of Theorem 4.2 with using $\left\{\theta_{\zeta_{m_{i}}}\right\}$ for $i \geq k+2$, there is a natural coalgebra decomposition

$$
D^{k}(V) \cong C^{m_{k+1}}(V) \otimes D^{k+1}(V) .
$$

In brief, the first construction of $E^{m_{k+1}}(1)=\operatorname{colim}_{g} T$ is the colimit of the map $g$ given by the composite

$$
T \longrightarrow D^{k} \longrightarrow T \stackrel{\theta_{\zeta_{k+2}}}{\longrightarrow} T
$$

and the inductive construction follows the lines in the proof of Theorem 4.2 with pre-composing $T \rightarrow D^{k} \hookrightarrow T$. This gives a monotone decreasing sequence of natural coalgebra retracts $C^{m_{k+1}}(i)$ of $D^{k}$ for $i=1,2, \ldots$ and the resulting natural coalgebra retract $C^{m_{k+1}}=\bigcap_{i=1}^{\infty} C^{m_{k+1}}(i)$ of $D^{k}$ has the property in primitives that $P_{n} C^{m_{k+1}}=0$ if $n$ is divisible by one of $m_{k+2}, m_{k+3}, \ldots$ and

$$
P_{n} C^{m_{k+1}}=P_{n} D^{k}
$$

if $n$ is not divisible by any of $m_{i}$ with $i \geq k+2$. Together with the fact that $P_{n} D=0$ if $n=m_{i} p^{r}$ for some $0 \leq i \leq k$ and $r \geq 0$ and $P_{n} D=P_{n} T$ otherwise, we have $P_{n} C^{m_{k+1}}=P_{n} T$ if $n=m_{k+1} p^{r}$ for some $r \geq 0$ and $P_{n} C^{m_{k+1}}=0$ otherwise. The induction is finished.

Now decomposition 4.6 induces a commutative diagram

$$
T \cong\left(\bigotimes_{i=0}^{k} C^{m_{i}}\right) \otimes D^{k} \cong\left(\bigotimes_{i=0}^{k+1} C^{m_{i}}\right) \otimes D^{k+1} \underset{\text { proj. }}{\stackrel{q_{k+1}}{\longrightarrow}} \bigotimes_{i=0}^{k+1} C^{m_{i}}
$$

that induces a coalgebra natural transformation

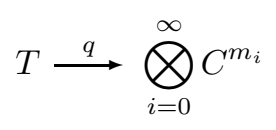

which is an isomorphism because, for each $n, D_{n}^{k}=0$ for sufficiently large $k>>0$. We finish the proof. 


\section{Proof of Theorem 1.1}

In this section, the ground ring is a field $\mathbf{k}$ of characteristic $p$.

Lemma 5.1. Let $Q$ be a $T_{n}$-projective sub functor of $L_{n}^{\text {res }}$. Then $Q$ is a subfunctor of $L_{n}$ and the sub Hopf algebra $T(Q(V))$ of $T(V)$ generated by $Q(V)$ is a natural coalgebra retract of $T(V)$.

Proof. It is easy to see that $\gamma_{n}\left(L_{n}^{\mathrm{res}}\right)=\gamma_{n}\left(L_{n}\right)=\operatorname{Lie}(n)$. By Proposition 3.1, the image of the natural transformation

$$
\Phi_{V}^{L_{n}^{\text {res }}}: \gamma_{n}\left(L_{n}^{\mathrm{res}}\right) \otimes_{\mathbf{k}\left(\Sigma_{n}\right)} V^{\otimes n} \longrightarrow L_{n}^{\mathrm{res}}(V)
$$

is $L_{n}(V)$. Since $Q$ is $T_{n}$-projective,

$$
\Phi_{V}^{Q}: \gamma_{n}(Q) \otimes_{\mathbf{k}\left(\Sigma_{n}\right)} V^{\otimes n} \longrightarrow Q(V)
$$

is an isomorphism by Proposition 2.10 (1). From the commutative diagram

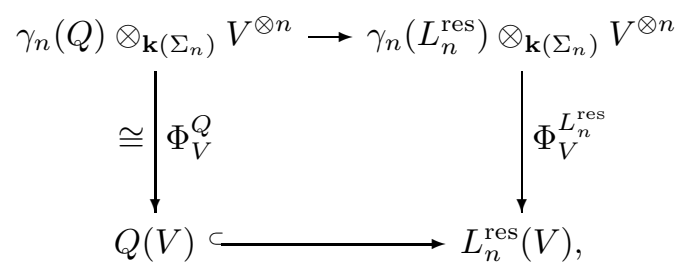

we have $Q \subseteq L_{n}$. By Proposition 2.10(4), there is a natural linear transformation

$$
r_{V}: T_{n}(V)=V^{\otimes n} \longrightarrow Q(V)
$$

with $\left.r_{V}\right|_{Q(V)}=\operatorname{id}_{Q(V)}$. Let

$$
H_{n}: T(V) \longrightarrow T\left(V^{\otimes n}\right)
$$

be the algebraic James-Hopf map induced by the geometric James-Hopf map by taking homology. Then there is a commutative diagram

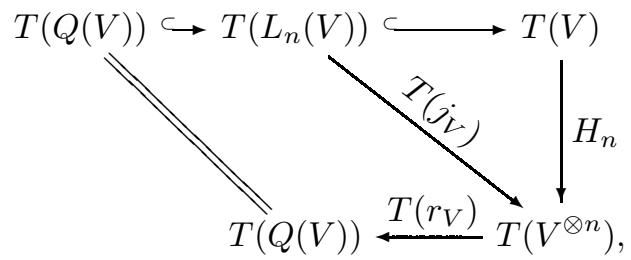

where the maps in the top row are the inclusions of sub Hopf algebras, $j_{V}$ is the canonical inclusion and the right triangle commutes by the geometric theorem in [28, Theorem 1.1]. Thus the sub Hopf algebra $T(Q(V))$ of $T(V)$ admits a natural coalgebra retraction and hence the result.

A natural sub Hopf algebra $B(V)$ of $T(V)$ is called coalgebra-split if the inclusion $B(V) \rightarrow T(V)$ admits a natural coalgebra retraction. For a Hopf algebra $A$, denote by $Q A$ the set of indecomposable elements of $A$. Let $I A$ be the augmentation ideal of $A$. If $B(V)$ is a natural sub Hopf algebra of $T(V)$, then there is a natural epimorphism $I B(V) \rightarrow Q B(V)$. Let $Q_{n} B(V)$ be the quotient of $B_{n}(V)=I B(V) \cap$ $T_{n}(V)$ in $Q B(V)$.

Theorem 5.2. Let $B(V)$ be a natural sub Hopf algebra of $T(V)$. Then the following statements are equivalent to each other:

1) $B(V)$ is a natural coalgebra-split sub Hopf algebra of $T(V)$. 
2) There is a natural linear transformation $r: T(V) \rightarrow B(V)$ such that $\left.r\right|_{B(V)}$ is the identity.

3) Each $Q_{n} B$ is naturally equivalent to a $T_{n}$-projective sub functor of $L_{n}$.

4) Each $Q_{n} B$ is a $T_{n}$-projective functor.

Proof. (1) $\Longrightarrow(2)$ and (3) $\Longrightarrow(4)$ are obvious. By [22, Theorem 8.6], (2) $\Longrightarrow(1)$. Thus $(1) \Longleftrightarrow(2)$. From the proof of $[22$, Theorem 8.8], (2) $\Longrightarrow(3)$.

$(4) \Longrightarrow(2)$. Since $B(V)$ is a sub Hopf algebra of primitively generated Hopf algebra $T(V), B(V)$ is primitively generated and so

$$
r_{n}: P_{n} B(V)=B(V) \cap L_{n}^{\mathrm{res}}(V) \longrightarrow Q_{n} B(V)
$$

is a natural epimorphism, where $L^{\text {res }}(V)=P T(V)$ is the free restricted Lie algebra generated by $V$. Since $Q_{n} B$ is $T_{n}$-projective, the map $r_{n}$ admits a natural crosssection $s_{n}: Q_{n} B(V) \hookrightarrow P_{n} B(V)$ by Proposition 2.10 (4).

Now we show that the inclusion $B(V) \rightarrow T(V)$ admits a natural linear retraction. By identifying $Q_{n} B(V)$ with $s_{n}\left(Q_{n} B(V)\right)$, we have

$$
B(V)=T\left(\bigoplus_{k=1}^{\infty} Q_{k} B(V)\right) \subseteq T(V) .
$$

Since each $Q_{k} B$ is a retract of the functor $T_{k}$,

$$
Q_{i_{1}} B \otimes \cdots \otimes Q_{i_{t}} B
$$

is a retract of $T_{i_{1}+i_{2}+\cdots+i_{t}}$ for any sequence $\left(i_{1}, \ldots, i_{t}\right)$. Note that $\left\{Q_{i} B(V) \mid i \geq 1\right\}$ are algebraically independent. Thus the summation

$$
\sum_{i_{1}+i_{2}+\cdots+i_{t}=q} Q_{i_{1}} B(V) \otimes Q_{i_{2}} B(V) \otimes \cdots \otimes Q_{i_{t}} B(V) \subseteq T_{q}(V)=V^{\otimes q} .
$$

is a direct sum. From the fact that

$$
\bigoplus_{i_{1}+i_{2}+\cdots+i_{t}=q} Q_{i_{1}} B \otimes Q_{i_{2}} B \otimes \cdots \otimes Q_{i_{t}} B
$$

is $T_{n}$-projective, there is natural linear retraction

$$
V^{\otimes q} \longrightarrow \bigoplus_{i_{1}+i_{2}+\cdots+i_{t}=q} Q_{i_{1}} B(V) \otimes Q_{i_{2}} B(V) \otimes \cdots \otimes Q_{i_{t}} B(V)
$$

for any $q \geq 1$. Hence the inclusion $B(V) \rightarrow T(V)$ admits a natural linear retraction.

Proof of Theorem 1.1. Let $B(V)$ be the sub Hopf algebra of $T(V)$ generated by

$$
L_{m_{i} p^{r}}(V) \quad \text { for } i \in I, r \geq 0 \text {. }
$$

Let $\left\{n_{j}\right\}_{j \geq 1}=\left\{m_{i} p^{r} \mid i \geq 1, r \geq 0\right\}$ with

$$
n_{1}=m_{1}<n_{2}<\cdots .
$$

Namely we rewrite the integers $m_{i} p^{r}$ in order. Let $B[k](V)$ be the sub Hopf algebra of $V$ generated by $L_{n_{j}}(V)$ for $1 \leq j \leq k$. By Theorem [5.2. it suffices to show that $Q_{n} B$ is $T_{n}$-projective for $n \geq 1$. Let $n$ be a fixed positive integer. Choose $k$ such that $n_{k} \geq n$. Then the inclusion $B[k] \hookrightarrow B$ induces an isomorphism

$$
Q_{n} B[k] \cong Q_{n} B
$$

because $B[k]$ and $B$ has the same set of generators in tensor length $\leq n$. Thus it suffices to show the following statement: 
For each $k \geq 1, B[k]$ is coalgebra-split.

The proof of this statement is given by induction on $k$. The statement holds for $k=1$ by Lemma 5.1 because $L_{m_{1}}$ is $T_{m_{1}}$-projective by 22, Corollary 6.7] from the assumption that $m_{i}$ is prime to $p$. Suppose that $B[k-1]$ is coalgebra-split. Thus there is a coalgebra natural transformation $r: T \rightarrow B[k-1]$ such that $\left.r\right|_{B[k-1]}$ is the identity map. Let $n_{k}=m_{i} p^{r}$ for some $i$ and $r$. Let $C^{m_{i}}$ be the natural coalgebra retract in Theorem 4.3 with a natural coalgebra retraction $r_{C}: T \rightarrow C^{m_{i}}$. Define $f: T \rightarrow T$ to be the composite

$$
T \stackrel{r_{C}}{\longrightarrow} C^{m_{i}} \longrightarrow T \stackrel{r}{\longrightarrow} B[k-1] \hookrightarrow T .
$$

Let $\tilde{E}=\operatorname{colim}_{f} T$ be the colimit with the canonical map

$$
q: T \longrightarrow \tilde{E} .
$$

As in lines of the proof of Theorem 4.2. there exists a coalgebra subfunctor $\tilde{C}$ of $C^{m_{i}}$ such that

$$
\left.q\right|_{\tilde{C}}: \tilde{C} \longrightarrow \tilde{E}
$$

is an isomorphism by [22, Theorem 4.5] with a natural coalgebra decomposition

$$
C^{m_{i}} \cong \tilde{C} \otimes \tilde{D} \text {. }
$$

According to [22, Lemma 5.3], the subfunctor $\tilde{D}$ of $C^{m_{i}}$ can be chosen as the cotensor product $\mathbf{k} \square_{\tilde{E}} C^{m_{i}}$ under the coalgebra map

$$
\left.q\right|_{C^{m_{i}}}: C^{m_{i}} \longrightarrow \tilde{E}
$$

and so there is a left exact sequence

$$
P_{n} \tilde{D} \hookrightarrow P_{n} C^{m_{i}} \stackrel{\left.P_{n} q\right|_{C} ^{m_{i}}}{\longrightarrow} \tilde{E}
$$

for any $n$.

By restricting the map $f$ as the composite in equation (5.1) to the primitives, we have the map

$$
P_{n} f: P_{n} T \stackrel{P_{n} r_{C}}{\longrightarrow} P_{n} C^{m_{i}} \longrightarrow P_{n} T \stackrel{P_{n} r}{\longrightarrow} P_{n} B[k-1] \hookrightarrow P_{n} T .
$$

If $n \neq m_{i} p^{t}$ for $t \geq 0$, then $P_{n} f=0$ because $P_{n} C^{m_{i}}=0$. If $n=m_{i} p^{t}$ for some $t \geq 0$ with $n<n_{k}$, then $P_{n} f$ is the identity map because $P_{n} C^{m_{i}}=P_{n} T$ and $P_{n} B[k-1]=P_{n} T=L_{n}^{\text {res }}$ as the sub Hopf algebra $B[k-1]$ contains $L_{m_{i} p^{s}}$ for $s \geq 0$. Thus

$$
P_{n} \tilde{C}=P_{n} C^{m_{i}}
$$

for $n<n_{k}$. From decomposition (5.2), we have

$$
P_{n} C^{m_{i}}=P_{n} \tilde{C} \oplus P_{n} \tilde{D}
$$

for all $n$ and so $P_{n} \tilde{D}=0$ for $n<n_{k}$. It follows that $\tilde{D}_{n}=0$ for $0<n<n_{k}$ and

$$
\tilde{D}_{n_{k}}=P_{n_{k}} \tilde{D} \text {. }
$$

Now consider the case $P_{n} f$ for $n=n_{k}=m_{i} p^{r}$. Since $P_{n} C^{m_{i}}=P_{n} T, P_{n} r_{C}=$ id and so $P_{n} f \circ P_{n} f=P_{n} f$ with

$$
P_{n} f(\alpha)=P_{n} r(\alpha)
$$

for $\alpha \in P_{n} T$. Thus the composite

$$
\operatorname{Im}\left(P_{n} f\right)=P_{n} B[k-1] \stackrel{\left.P_{n} q\right|_{B[k-1]}}{\longrightarrow} P_{n} \tilde{E}=\operatorname{colim}_{P_{n} f} P_{n} T
$$


is an isomorphism. From exact sequence (5.3), we have

$$
P_{n} \tilde{D}=\operatorname{Ker}\left(P_{n} r: P_{n} C^{m_{i}}=P_{n} T \rightarrow P_{n} B[k-1]\right) .
$$

Let $j: P_{n} B[k-1] \hookrightarrow P_{n} C^{m_{i}}=P_{n} T$ be the inclusion. From the commutative diagram

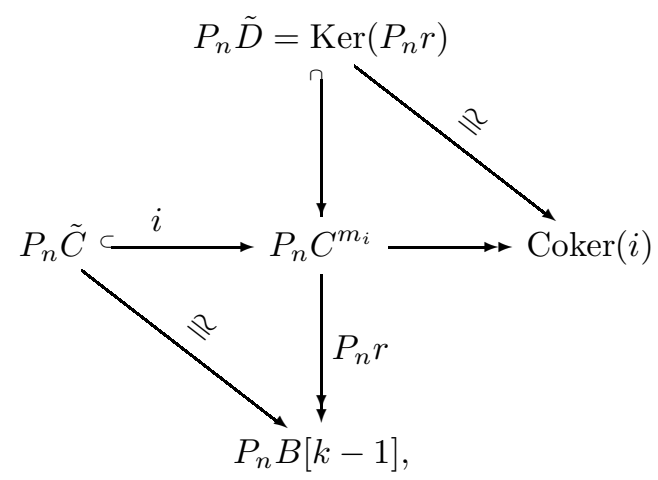

the summation $P_{n} B[k-1]+P_{n} \tilde{D}$ in $P_{n} T=P_{n} C^{m_{i}}$ is a direct sum with the decomposition

$$
P_{n} C^{m_{i}}=P_{n} T=P_{n} B[k-1] \oplus P_{n} \tilde{D} .
$$

From definition of $B[k], P B[k](V)$ is the restricted sub Lie algebra of $L^{\text {res }}(V)=$ $P T(V)$ generated by $L_{n_{i}}(V)$ for $1 \leq i \leq k$. It follows that $P_{n} B[k]=L_{n}^{\text {res }}=P_{n} T=$ $P_{n} C^{m_{i}}$ and

$$
Q_{n} B[k] \cong P_{n} B[k] / P_{n} B[k-1]=P_{n} C^{m_{i}} / P_{n} B[k-1] \cong P_{n} \tilde{D} .
$$

From decomposition (5.2), $\tilde{D}_{n}$ is a natural summand of $C_{n}^{\min }$. Since $C^{\text {min }}$ is a coalgebra retract of $T, C_{n}^{\min }$ is a natural summand of $T_{n}$. Thus $\tilde{D}_{n}$ is $T_{n}$-projective. By identity (5.5), $P_{n} \tilde{D}$ is $T_{n}$-projective. Thus $Q_{n} B[k]$ is $T_{n}$-projective. By Theorem [5.2, $B[k]$ is coalgebra-split. The induction is finished and hence the result.

By inspecting the proof, we have the following slightly stronger statement.

Theorem 5.3. Let $\mathcal{M}=\left\{m_{i}\right\}_{i \in I}$ be finite or infinite set of positive integers prime to $p$ with each $m_{i}>1$. Let $f: I \rightarrow\{0,1,2, \ldots\} \cup\{\infty\}$ be a function. Then the sub Hopf algebra $B^{\mathcal{M}, f}(V)$ of $T(V)$ generated by

$$
L_{m_{i} p^{r}}(V) \quad \text { for } i \in I, 0 \leq r<f(i)
$$

is natural coalgebra-split.

\section{Decompositions of Lie Powers}

Let $m=k p^{r}$ with $k \not \equiv 0 \bmod p$ and $k>1$. According to [22, Theorem 10.7], the functor $L_{k p^{r}}$ admits the following functorial decomposition:

$$
L_{k p^{r}}=L_{k p^{r}}^{\prime} \oplus L_{p}\left(L_{k p^{r-1}}^{\prime}\right) \oplus \cdots \oplus L_{p^{r}}\left(L_{k}^{\prime}\right)
$$

for each $r \geq 0$ starting with $L_{k}^{\prime}=L_{k}$, where each $L_{k p^{r}}^{\prime}$ is a summand of $T_{k p^{r}}$ which is called $T_{k p^{r}}$-projective in our terminology. By evaluating on $V$, one gets a decomposition of the $\mathbf{k}(\mathrm{GL}(V))$-module $L_{k p^{r}}(V)$ given [3, Theorem 4.4] using a different approach from representation theory, where $L_{k p^{r}}^{\prime}(V)$ was denoted by $B_{k p^{r}}$ in [3. From Theorem 5.3, we can obtain various new decompositions of $L_{k p^{r}}$ and so the decompositions of the $\mathbf{k}(\mathrm{GL}(V))$-module $L_{k p^{r}}(V)$ by evaluating on $V$. 
Let $\mathcal{M}=\left\{m_{i}\right\}_{i \in I}$ be finite or infinite set of positive integers prime to $p$ with each $m_{i}>1$. Let $f: I \rightarrow\{0,1,2, \ldots\} \cup\{\infty\}$ be a function. Let $B^{\mathcal{M}, f}(V)$ be the sub Hopf algebra of $T(V)$ generated by

$$
L_{m_{i} p^{r}}(V) \quad \text { for } i \in I, 0 \leq r<f(i) .
$$

According to Theorem [5.3, $B^{\mathcal{M}, f}$ is coalgebra-split and so $Q_{n} B^{\mathcal{M}, f}$ is $T_{n}$-projective by Theorem 5.2. Since $B^{\mathcal{M}, f}(V)$ is sub Hopf algebra of the primitively generated Hopf algebra $T(V)$, it is primitively generated by [18, Proposition 6.13] and so there is a natural epimorphism

$$
\phi_{n}: P_{n} B^{\mathcal{M}, f} \rightarrow Q_{n} B^{\mathcal{M}, f} .
$$

From Proposition 2.10(4), the map $\phi_{n}$ admits a natural cross-section because $Q_{n} B^{\mathcal{M}, f}$ is $T_{n}$-projective. Thus there is a subfunctor $D_{n}^{\mathcal{M}, f}$ of $P_{n} B^{\mathcal{M}, f}$ such that

$$
\phi_{n} \mid: D_{n}^{\mathcal{M}, f} \rightarrow Q_{n} B^{\mathcal{M}, f}
$$

is a natural isomorphism. Since $D_{n}^{\mathcal{M}, f}$ is $T_{n}$-projective,

$$
D_{n}^{\mathcal{M}, f} \subseteq P_{n} B^{\mathcal{M}, f} \cap L_{n}
$$

from the lines in the proof of Lemma 5.1. Thus $D_{n}^{\mathcal{M}, f}$ is a $T_{n}$-projective subfunctor of $L_{n}$. From the fact that $D^{\mathcal{M}, f} \cong Q_{n} B^{\mathcal{M}, f}$ and $B^{\mathcal{M}, f}$ is isomorphic to the tensor algebra generated by $Q_{n} B^{\mathcal{M}, f}$ with $n \geq 1$, the inclusion

$$
\bigoplus_{n=1}^{\infty} D_{n} \hookrightarrow B^{\mathcal{M}, f}
$$

induces a natural isomorphism

$$
T\left(\bigoplus_{n=1}^{\infty} D_{n}\right) \cong B^{\mathcal{M}, f}
$$

Since the algebra $B^{\mathcal{M}, f}$ is generated by $L_{m_{i} p^{r}}(V)$ for $m_{i} \in \mathcal{M}$ and $0 \leq r<f(i)$, we have

$$
D_{n}^{\mathcal{M}, f}=0 \text { if } n \neq m_{i} p^{r} \text { for some } m_{i} \in \mathcal{M} \text { and some } 0 \leq r<f(i) .
$$

Let $\left\{m_{i} p^{r} \mid m_{i} \in \mathcal{M}, 0 \leq r<f(i)\right\}=\left\{n_{1}, n_{2}, \ldots\right\}$ with $n_{1}<n_{2}<\cdots$ and let $\alpha$ be the cardinality of the set $\left\{m_{i} p^{r} \mid m_{i} \in \mathcal{M}, 0 \leq r<f(i)\right\}$. Then decomposition (6.1) becomes

$$
T\left(\bigoplus_{i=1}^{\alpha} D_{n_{i}}\right) \cong B^{\mathcal{M}, f} .
$$

and so a natural isomorphism

$$
P T\left(\bigoplus_{i=1}^{\alpha} D_{n_{i}}\right)=L^{\mathrm{res}}\left(\bigoplus_{i=1}^{\alpha} D_{n_{i}}\right) \cong P B^{\mathcal{M}, f}=B^{\mathcal{M}, f} \cap L^{\mathrm{res}} .
$$

According to Proposition 4.1 the sub Hopf algebra $B^{\mathcal{M}, f}$ is also a natural coalgebra retract of $T$ if we change the ground ring $R$ to be $p$-local integers. Notice that $P T=L$ and $P B^{\mathcal{M}, f}=B^{\mathcal{M}, f} \cap L$ when $R=\mathbb{Z}_{(p)}$. By changing the ground ring back to $\mathbb{Z} / p$ and then extending it to $\mathbf{k}$, we have the following decomposition:

$$
L\left(\bigoplus_{i=1}^{\alpha} D_{n_{i}}\right) \cong B^{\mathcal{M}, f} \cap L .
$$


For applying the Hilton-Milnor Theorem to determine $B^{\mathcal{M}, f} \cap L_{n}$, let us recall the terminology of basic product from [27, p.512]. Let $x_{1}, \ldots, x_{k}$ be letters. A monomial means a formal product $w=x_{i_{1}} x_{i_{2}} \cdots x_{i_{n}}$ with $1 \leq i_{1}, \ldots, i_{t} \leq k$, where the word length $n$ is called the weight of $w$. We define the basic products of weight $n$, by induction on $n$; and for each such product, a non-negative integer $r(w)$, called its rank. These are to be linearly ordered, in such a way that $w_{1}<w_{2}$ if the weight of $w_{1}$ is less than the weight of $w_{2}$. The serial number $s(w)$ is the number of basic products $\leq w$ in term of this ordering. The basic products of weight 1 are the letters $x_{1}, \ldots, x_{k}$ with the order $x_{1}<x_{2}<\cdots<x_{k}$. The rank $r\left(x_{i}\right)=0$ and the serial number $s\left(x_{i}\right)=i$. Suppose that the basic products of weight less than $n$ have been defined and linearly ordered in such a way that $w_{1}<w_{2}$ if the weight of $w_{1}$ is less than that of $w_{2}$; and suppose that the rank $r(w)$ of such a product has been defined. Then the basic products of weight $n$ are all monomials of the $w_{1} w_{2}$ of weight $n$, for which $w_{1}$ and $w_{2}$ are basic products, $w_{2}<w_{1}$ and $r\left(w_{1}\right) \leq s\left(w_{2}\right)$. Give these an arbitrary linear order, and define $r\left(w_{1} w_{2}\right)=s\left(w_{2}\right)$.

Let $\mathcal{W}_{k}$ be the set of all basic products on the letters $x_{1}, \ldots, x_{k}$ by forgetting the ordering. Then

for each $k$. Let

$$
\mathcal{W}_{k} \subseteq \mathcal{W}_{k+1}
$$

$$
\mathcal{W}_{\infty}=\bigcup_{k=1}^{\infty} \mathcal{W}_{k}
$$

The elements in $\mathcal{W}$ are called basic products on the sequence of the letters $x_{i}$ for $i \geq 1$. For each basic product $w=x_{i_{1}} \cdots x_{i_{t}} \in \mathcal{W}_{\alpha}$, define

$$
w\left(D^{\mathcal{M}, f}\right)=D_{n_{i_{1}}}^{\mathcal{M}, f} \otimes \cdots \otimes D_{n_{i_{t}}}^{\mathcal{M}, f}
$$

with the tensor length with respect to $D^{\mathcal{M}, f}$

$$
d(w)=n_{i_{1}}+n_{i_{2}}+\cdots+n_{i_{t}}
$$

and the natural transformation

$$
\phi_{w}: w\left(D^{\mathcal{M}, f}\right)(V)=\longrightarrow T\left(\bigoplus_{i=1}^{\alpha} D_{n_{i}}(V)\right) \cong B^{\mathcal{M}, f}(V)
$$

given by

$$
\phi_{w}\left(z_{1} \otimes z_{2} \otimes \cdots \otimes z_{t}\right)=\left[\left[z_{1}, z_{2}, \ldots, z_{t}\right]\right.
$$

for $z_{j} \in D_{n_{i_{j}}}^{\mathcal{M}, f}(V)$. Then the map $\phi_{w}$ extends uniquely to a natural transformation of Hopf algebras

$$
T \phi_{w}: T\left(w\left(D^{\mathcal{M}, f}\right)\right) \longrightarrow T\left(\bigoplus_{i=1}^{\alpha} D_{n_{i}}(V)\right) \cong B^{\mathcal{M}, f}(V)
$$

by the universal property of tensor algebras. Now by taking the homology from the Hilton-Milnor Theorem [27. Theorem 6.7], we have the natural isomorphism of coalgebras

$$
\theta: \bigotimes_{w} T\left(w\left(D^{\mathcal{M}, f}\right)\right) \stackrel{\cong}{\longrightarrow} T\left(\bigoplus_{i=1}^{\alpha} D_{n_{i}}\right) \cong B^{\mathcal{M}, f},
$$

where $w$ runs over all basic products in $\mathcal{W}_{\alpha}$, the tensor product is linearly ordered and the natural transformation $\theta$ is given by the ordered product of $T \phi_{w}$ which is 
well-defined because the tensor length $d(w)$ tends to $\infty$ as the weight of $w$ tends to $\infty$. By restricting to Lie powers, we have the decomposition

$$
\theta \mid: \bigotimes_{w} L\left(w\left(D^{\mathcal{M}, f}\right)\right) \stackrel{\cong}{\longrightarrow}\left(\bigoplus_{i=1}^{\alpha} D_{n_{i}}\right) \cong B^{\mathcal{M}, f} \cap L .
$$

By taking tensor length, we obtain the following decomposition theorem.

Theorem 6.1. Let $\mathcal{M}=\left\{m_{i}\right\}_{i \in I}$ be finite or infinite set of positive integers prime to $p$ with each $m_{i}>1$ and let $f: I \rightarrow\{0,1,2, \ldots\} \cup\{\infty\}$ be a function. Then

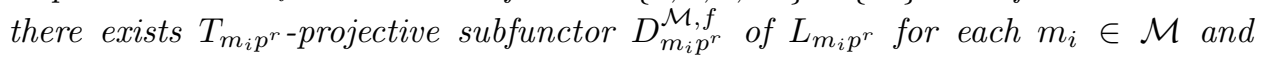
$0 \leq r<f(i)$ such that

$$
L_{m_{i} p^{r}}=\bigoplus_{d(w) \mid m_{i} p^{r}} L_{m_{i} p^{r} / d(w)}\left(w\left(D^{\mathcal{M}, f}\right)\right)
$$

for $m_{i} \in \mathcal{M}$ and $0 \leq r<f(i)$, where $w$ runs over basic products with $d(w) \mid m_{i} p^{r}$.

Note. Since each $D_{n_{i}}$ is $T_{n_{i}}$-projective, the tensor product $w\left(D^{\mathcal{M}, f}\right)$ is $T_{d(w)^{-}}$ projective. If $m_{i} p^{r} / d(w)$ is prime to $p$, then the Lie power $L_{m_{i} p^{r} / d(w)}\left(w\left(D^{\mathcal{M}, f}\right)\right)$ is $T_{m_{i} p^{r}}$-projective. Thus the non $T_{m_{i} p^{r}}$-projective summands of $L_{m_{i} p^{r}}$ occurs in the those factors $L_{m_{i} p^{r} / d(w)}\left(w\left(D^{\mathcal{M}, f}\right)\right)$ with $m_{i} p^{r} / d(w) \equiv 0 \bmod p$.

Another remark is that the multiplicity of each factor $L_{m_{i} p^{r} / d(w)}\left(w\left(D^{\mathcal{M}, f}\right)\right)$ can be determined as follows: Let $w$ be a basic product involving the letters $x_{j_{1}}, \ldots, x_{j_{k}}$ such that $x_{j_{i}}$ occurs $l_{i}$ times and $d(w) \mid m_{i} p^{r}$. According to [27, (6.4), p.514], the multiplicity of the factor $L_{m_{i} p^{r} / d(w)}\left(w\left(D^{\mathcal{M}, f}\right)\right)$ is given by the formula

$$
\frac{1}{l} \sum_{d \mid l_{0}} \mu(d) \frac{\left(\frac{l}{d}\right) !}{\left(\frac{l_{1}}{d}\right) ! \cdots\left(\frac{l_{k}}{d}\right) !}
$$

where $\mu$ is the Möbius function, $l_{0}$ is the greatest common divisor of $l_{1}, \ldots, l_{k}$ and $l=l_{1}+\cdots+l_{k}$.

Example 6.2. Let $\mathbf{k}$ be of characteristic 2. Let $\mathcal{M}=\left\{m_{1}=3\right\}$ and let $f(1)=3$. Then we have natural coalgebra-split sub Hopf algebra

$$
B^{\mathcal{M}, f}(V)=\left\langle L_{3}(V), L_{6}(V), L_{12}(V),\right\rangle
$$

of $T(V)$ with $D_{3}^{\mathcal{M}, f}=L_{3}, D_{6}^{\mathcal{M}, f} \cong L_{6}^{\prime}=L_{6} / L_{2}\left(L_{3}\right)$ and

$$
D_{12}^{\mathcal{M}, f} \cong L_{12} /\left(\left[L_{6}^{\prime}, L_{6}^{\prime}\right] \oplus\left[\left[L_{6}^{\prime}, L_{3}\right], L_{3}\right] \oplus L_{4}\left(L_{3}\right)\right)
$$

From Theorem 6.1, we have the decomposition

$$
\begin{aligned}
L_{12} & =D_{12} \oplus L\left(D_{3} \oplus D_{6}\right) \cap L_{12} \\
& =D_{12} \oplus L_{4}\left(D_{3}\right) \oplus L_{2}\left(D_{6}\right) \oplus\left[\left[D_{6}, D_{3}\right], D_{3}\right] \\
& \cong D_{12} \oplus L_{4}\left(L_{3}\right) \oplus L_{2}\left(L_{6}^{\prime}\right) \oplus\left[\left[L_{6}^{\prime}, L_{3}\right], L_{3}\right] .
\end{aligned}
$$

By comparing with [22, Theorem 10.7] or [3, Theorem 4.4], the $T_{12}$-projective summand

$$
\left[\left[L_{6}^{\prime}, L_{3}\right], L_{3}\right] \cong L_{6}^{\prime} \otimes L_{3} \otimes L_{3}
$$

can be recognized in our decomposition for $L_{12}$. 
Let $\mathcal{M}$ be the set of all positive integers $m_{i}$ with $m_{i}$ prime to $p$ and $m_{i}>1$ and let $f(i)=\infty$ for all $i$. Then the set

$$
\left\{m_{i} p^{r} \mid m_{i} \in \mathcal{M} r \geq 0\right\}=\mathbb{N} \backslash\left\{1, p, p^{2}, p^{3}, \ldots\right\} .
$$

Let

$$
\bar{D}_{n}=D_{n}^{\mathcal{M}, f}
$$

for $n$ not a power of $p$. As a special case of Theorem 6.1 we have the following:

Corollary 6.3. There exists $T_{n}$-projective subfunctor $\bar{D}_{n}$ of $L_{n}$ for each $n$ not a power of $p$ such that

$$
L_{m}=\bigoplus_{d(w) \mid m} L_{m / d(w)}(w(\bar{D}))
$$

for any $m$ not a power of $p$, where $w$ runs over basic products with $d(w) \mid m$.

\section{REFERENCES}

[1] K. Akin, D. A. Buchsbaum and J. Weyman, Schur functors and Schur complexes, Advances in Mathematics 44 (1982), 207-278.

[2] R. M. Bryant and M. Johnson, Lie powers and Witt vectors, J. Algebr. Comb. 28 (2008), 169-187.

[3] R. M. Bryant and M. Schocker, The decomposition of Lie powers, Proc. London Math. Soc. 93(2006), 175-196.

[4] R. M. Bryant and R. Stöhr, Lie powers in prime degree, Quart. J. Math. 56 (2005), 473-489.

[5] F. Cohen, On combinatorial group theory in homotopy, Contemp. Math. 188 (1995), 57-63.

[6] F. R. Cohen, J. C. Moore and J. A. Neisendorfer, Torsion in homotopy groups, Ann. of Math. (2) 109 (1979), 121-168.

[7] Curtis and Reiner, Methods of Representation Theory, Vol. I, Pure and Applied Mathematics Wiley (1981).

[8] S. Donkin, The q-Schur algebra, Lecture Notes Series Volumn 253, London Mathematical Society, 1998.

[9] S. Donkin and K. Erdmann, Tilting modules, symmetric functions, and the module structure of the free Lie algebra, J. of Algebra 203 (1998) 69-90.

[10] K. Erdmann and M. Schocker, Modular Lie powers and the Solomon descent algebra, Math. Z. 253 (2006), 295-313.

[11] J. A. Green, Polynomial representation of $\mathrm{GL}_{n}$, Lecture Notes in Mathematics 830 (1980), Springer-Verlag, Berlin/Heidelberg/New York.

[12] J. Grbic, P. S. Selick and J. Wu, The decomposition of the loop space of the mod 2 Moore space, Algebraic and Geometric Topology 8 (2008), 945-951.

[13] J. Grbic and J. Wu, Natural transformations of tensor algebras and representations of combinatorial groups, Algebraic and Geometric Topology 6 (2006), 2189-2228.

[14] G. James and A. Kerber, The representation theory of the symmetric groups, Encyclopedia of Mathematics and its Applications 16 (1981).

[15] Nick Kuhn, Generic representation theory of the finite general linear groups and the Steenrod algebra I, Amer. J. Math. 116 (1994), 327-360.

[16] Nick Kuhn, Generic representation theory of the finite general linear groups and the Steenrod algebra II, K-theory J. 8 (1994), 395-428.

[17] Nick Kuhn, Generic representation theory of the finite general linear groups and the Steenrod algebra III, K-theory J. 9 (1995), 273-303.

[18] J. Milnor and J. Moore, On the structure of Hopf Algebras, Ann. Math. 81 (1965), 211-264.

[19] I. Schur, Über eine Klasse von Matrizen, die sich einer gegebenen Matrix zourden lassen (1901), in I. Schur, Gesammelte Abhandlungen I, 1-70, Springer, Berlin, 1973.

[20] P. S. Selick, S. D. Theriault and J. Wu, Functorial decompositions of looped coassociative co-H spaces, Canadian J. Math. 58 (2006), 877-896. 
[21] P. S. Selick, S. D. Theriault and J. Wu, functorial homotopy decompositions of looped co-H-spaces, Math Z., to appear.

[22] P. S. Selick and J. Wu, On natural decompositions of loop suspensions and natural coalgebra decompositions of tensor algebras, Memoirs AMS 148 (2000), No. 701.

[23] P. S. Selick and J. Wu, On functorial decompositions of self-smash products, Manuscripta Math.111 (2003), 435-457.

[24] P. S. Selick and J. Wu, The functor $A^{\text {min }}$ on p-local spaces, Math. Z. 253(2006), 435-451.

[25] P. S. Selick and J. Wu, Some calculations of $\operatorname{Lie}^{\max }(n)$ for low $n$, J. of Pure and Appl. Algebra 212 (2008), 2570-2580.

[26] S. D. Theriault, Homotopy decompositions involving the loops of coassociative Co- $H$ spaces, Canad. J. Math. 55 (2003), 181-203.

[27] G. W. Whitehead, Elements of homotopy theory, Graduate Texts in Mathematics, Springer, Berlin (1978).

[28] J. Wu, On combinatorial calculations of the James-Hopf maps, Topology 37 (1998), 1011-1023.

[29] J. Wu, Homotopy theory of the suspensions of the projective plane, Memoirs AMS 162 (2003), No. 769.

[30] J. Wu, On maps from loop suspensions to loop spaces and the shuffle relations on the Cohen groups, Memoirs AMS, 180 (2006), No. 851.

[31] J. Wu, EHP sequences for (p-1)-cell complexes and the functor Amin, Israel J. Math. to appear.

Institute of Mathematics and Physics, Shijiazhuang Railway Institute, Shijiazhuang 050043, CHINA

E-mail address: yanjinglee@163.com

School of Mathematical Sciences, Dalian University of Technology, Dalian 116024, CHINA

E-mail address: fclei@dlut.edu.cn

Department of Mathematics, National University of Singapore, Block S17, 10, Lower Kent Ridge Road, Singapore 119076

E-mail address: matwuj@nus.edu.sg

URL: http://www.math.nus.edu.sg/ ${ }^{\sim m a t w u j i e ~}$ 